\title{
GEOMETRIC REALIZATION OF KHOVANOV-LAUDA-ROUQUIER ALGEBRAS ASSOCIATED WITH BORCHERDS-CARTAN DATA
}

\author{
SEOK-JIN KANG ${ }^{1}$, MASAKI KASHIWARA ${ }^{2}$, AND EUIYONG PARK ${ }^{3}$
}

\begin{abstract}
We construct a geometric realization of the Khovanov-Lauda-Rouquier algebra $R$ associated with a symmetric Borcherds-Cartan matrix $A=\left(a_{i j}\right)_{i, j \in I}$ via quiver varieties. As an application, if $a_{i i} \neq 0$ for any $i \in I$, we prove that there exists a 1-1 correspondence between Kashiwara's lower global basis (or Lusztig's canonical basis) of $U_{\mathbb{A}}^{-}(\mathfrak{g})\left(\right.$ resp. $V_{\mathbb{A}}(\lambda)$ ) and the set of isomorphism classes of indecomposable projective graded modules over $R$ $\left(\right.$ resp. $\left.R^{\lambda}\right)$.
\end{abstract}

\section{INTRODUCTION}

The Khovanov-Lauda-Rouquier algebras (or quiver Hecke algebras) were introduced independently by Khovanov-Lauda [13, 14] and Rouquier [18] to construct a categorification of quantum groups associated with symmetrizable Cartan data. For a dominant integral weight $\lambda \in \mathrm{P}^{+}$, Khovanov and Lauda conjectured that the cyclotomic quotient $R^{\lambda}$ of the KhovanovLauda-Rouquier algebra $R$ gives a categorification of the irreducible highest weight module $V(\lambda)[13]$. Recently, this conjecture was proved by Kang and Kashiwara [8]. In [21] Webster also gave a proof of this conjecture by a completely different method.

When the Cartan datum is symmetric, Varagnolo-Vasserot [20] and Rouquier [19] gave a geometric realization of Khovanov-Lauda-Rouquier algebras via quiver varieties and proved that the isomorphism classes of projective indecomposable modules correspond to Kashiwara's lower global basis (or Lusztig's canonical basis) [12, 15].

In [10], Kang, Oh and Park introduced a family of Khovanov-Lauda-Rouquier algebras $R$ associated with symmetrizable Borcherds-Cartan data and showed that they provide a categorification of quantum generalized Kac-Moody algebras and their crystals. More precisely, let $U_{\mathbb{A}}^{-}(\mathfrak{g})$ be the integral form of the negative half of the quantum generalized KacMoody algebra $U_{q}(\mathfrak{g})$ associated with a Borcherds-Cartan matrix $A=\left(a_{i j}\right)_{i, j \in I}$ and let us

2010 Mathematics Subject Classification. 14F05, 14F43 17B67, 81R10.

1 This work was supported by KRF Grant \# 2007-341-C00001 and NRF Grant \# 2010-0019516.

2 This work was supported by Grant-in-Aid for Scientific Research (B) 22340005, Japan Society for the Promotion of Science.

${ }^{3}$ This work was supported by JSPS Postdoctoral Fellowships for Foreign Researchers. 
set $K_{0}(R)=\bigoplus_{\alpha \in Q^{+}} K_{0}\left(R(\alpha)\right.$-pmod), where $K_{0}(R(\alpha)$-pmod) is the Grothendieck group of the category $R(\alpha)$-pmod of finitely generated projective graded $R(\alpha)$-modules. Then it was proved in [10] that there exists an injective bialgebra homomorphism

$$
\Phi: U_{\mathbb{A}}^{-}(\mathfrak{g}) \hookrightarrow K_{0}(R)
$$

and that $\Phi$ is an isomorphism when $a_{i i} \neq 0$ for any $i \in I$.

A big difference with the case of Kac-Moody algebras is that the defining relations of $R$ contain a family of polynomials $\mathcal{P}_{i}$ of degree $1-\frac{a_{i i}}{2}(i \in I)$ as twisting factors for commutation and braid relations. As we will see in Lemma 2.6, the polynomials $\mathcal{P}_{i}$ have a natural geometric interpretation.

For a dominant integral weight $\lambda \in \mathrm{P}^{+}$, if $a_{i i} \neq 0$ for any $i \in I$, it was proved in [9] that the cyclotomic quotient $R^{\lambda}$ of $R$ provides a categorification of the irreducible highest weight $U_{q}(\mathfrak{g})$-module $V(\lambda)$. That is, there is a $U_{\mathbb{A}}(\mathfrak{g})$-module isomorphism

$$
\Phi^{\lambda}: V_{\mathbb{A}}(\lambda) \stackrel{\sim}{\longrightarrow} K_{0}\left(R^{\lambda}\right):=\bigoplus_{\alpha \in Q^{+}} K_{0}\left(R^{\lambda}(\alpha) \text {-pmod }\right),
$$

where $K_{0}\left(R^{\lambda}(\alpha)\right.$-pmod) is the Grothendieck group of the category of finitely generated projective graded $R^{\lambda}(\alpha)$-modules.

In this paper, following the framework of [20], we construct a geometric realization of Khovanov-Lauda-Rouquier algebras associated with symmetric Borcherds-Cartan data via quivers possibly with loops. One of the main ingredients is Steinberg-type varieties arising from quivers. As an application, when $a_{i i} \neq 0$ for any $i \in I$, we prove that the isomorphism $\Phi$ (resp. $\Phi^{\lambda}$ ) gives a 1-1 correspondence between Kashiwara's lower global basis (or Lusztig's canonical basis) of $U_{\mathbb{A}}^{-}(\mathfrak{g})$ (resp. $V_{\mathbb{A}}(\lambda)$ ) given in [11] and the set of isomorphism classes of indecomposable projective $R$-modules (resp. indecomposable projective $R^{\lambda}$-modules).

Let us explain our results more precisely. Let $Q=(I, \Omega)$ be an arbitrary locally finite quiver with a vertex set $I$ and an oriented edge set $\Omega$. The edge set $\Omega$ may have loops which will give a geometric interpretation of the polynomials $\mathcal{P}_{i}$ in the definition of $R$.

Let $\alpha \in \mathrm{Q}^{+}$with $|\alpha|=m$ and $I^{\alpha}=\left\{\nu=\left(\nu_{1}, \ldots, \nu_{m}\right) \in I^{m} \mid \alpha=\alpha_{\nu_{1}}+\cdots+\alpha_{\nu_{m}}\right\}$. We fix an $I$-graded vector space $V_{\alpha}=\bigoplus_{i \in I} V_{i}$ with $\underline{\operatorname{dim}}\left(V_{\alpha}\right)=\alpha$. Let $\mathcal{E}_{\alpha}$ be the set of all representations of $Q$ with dimension vector $\alpha$, let $\mathcal{F}_{\nu}$ be the set of complete flags of type $\nu \in I^{\alpha}$, and let $\widetilde{\mathcal{F}}_{\nu}=\left\{(x, F) \in \mathcal{E}_{\alpha} \times \mathcal{F}_{\nu} \mid F\right.$ is strictly $x$-stable $\}$. Set

$$
\mathrm{G}_{\alpha}^{\Omega}=\mathrm{G}_{\alpha} \times \mathrm{H}^{\Omega}
$$

where $\mathrm{G}_{\alpha}=\prod_{i \in I} G L\left(V_{i}\right)$ and $\mathrm{H}^{\Omega}$ is the torus corresponding to the edge set $\Omega$ defined by

$$
\mathrm{H}^{\Omega}=\prod_{a \in \Omega} \mathbb{C}^{*}
$$


The group $\mathrm{G}_{\alpha}$ acts on $\mathcal{E}_{\alpha}$ by conjugation and transitively on $\mathcal{F}_{\nu}$, while $\mathrm{H}^{\Omega}$ acts on $\mathcal{E}_{\alpha}$ by multiplication and trivially on $\mathcal{F}_{\nu}$.

For $\nu, \nu^{\prime} \in I^{\alpha}$, we first define the Steinberg-type variety

$$
\mathcal{Z}_{\nu, \nu^{\prime}}=\widetilde{\mathcal{F}}_{\nu} \times \mathcal{E}_{\alpha} \widetilde{\mathcal{F}}_{\nu^{\prime}}
$$

and then consider the convolution algebra $\mathfrak{R}(\alpha)$ and its polynomial representation $\mathfrak{P o r}(\alpha)$ as follows:

$$
\mathfrak{R}(\alpha)=\bigoplus_{\nu, \nu^{\prime} \in I^{\alpha}} H_{*}^{\mathrm{G}_{\alpha}^{\Omega}}\left(\mathcal{Z}_{\nu, \nu^{\prime}}\right)\left\langle-2 \operatorname{dim}_{\mathbb{C}} \widetilde{\mathcal{F}}_{\nu}\right\rangle, \quad \mathfrak{P o l}(\alpha)=\bigoplus_{\nu \in I^{\alpha}} H_{\mathrm{G}_{\alpha}^{\Omega}}^{*}\left(\widetilde{\mathcal{F}}_{\nu}\right) .
$$

In Lemma 2.3, we investigate the properties of the filtration $\mathfrak{R} \leq w$ of $\mathfrak{R}(\alpha)$ given in (2.2.8), and in Lemma 2.6, using the commutative diagram (2.3.10), we compute the equivariant Euler classes of fixed points in $\widetilde{\mathcal{F}}_{\nu}$ and $\mathcal{Z}_{\nu, \nu^{\prime}}^{s_{j}}$, which will lead us to an explicit description of the $\mathfrak{R}(\alpha)$-action on $\mathfrak{P o l}(\alpha)$. Here, the polynomials $\mathcal{P}_{i}$ arise naturally from the computation of the Euler classes relative to the loops. In Proposition 2.7, we show that $\mathfrak{P o l}(\alpha)$ is a faithful polynomial representation and give an explicit description of $\mathfrak{R}(\alpha)$-action on $\mathfrak{P o l}(\alpha)$. It turns out that the cohomology ring

$$
\mathbf{H}=H_{\mathrm{H}^{\Omega}}^{*}(\mathrm{pt}) \simeq \bigotimes_{a \in \Omega} \mathbb{C}\left[\hbar_{a}\right]
$$

plays the role of a base ring in the definition of Khovanov-Lauda-Rouquier algebras. Using the faithful polynomial representation $\mathfrak{P o l}(\alpha)$, we finally prove that $\mathfrak{R}(\alpha)$ is isomorphic to the Khovanov-Lauda-Rouquier algebra $R(\alpha)$ (Theorem 2.8).

Furthermore, in Proposition 3.1, we investigate the relation between the category $R(\alpha)$ pmod and the full subcategory $\mathcal{Q}_{\alpha}$ of $\mathbf{D}_{\mathrm{G}_{\alpha}^{\Omega}}^{b}\left(\mathcal{E}_{\alpha}\right)$. In Theorem 3.2 and Corollary 3.3, we show that, when $a_{i i} \neq 0$ for any $i$, the isomorphism $\Phi$ (resp. $\Phi^{\lambda}$ ) gives a 1-1 correspondence between Kashiwara's lower global basis (or Lusztig's canonical basis) of $U_{\mathbb{A}}^{-}(\mathfrak{g})$ (resp. $V_{\mathbb{A}}(\lambda)$ ) given in [11] and the set of isomorphism classes of indecomposable projective $R$-modules (resp. indecomposable projective $R^{\lambda}$-modules).

\section{Khovanov-Lauda-Rouquier algebras}

1.1. Quantum generalized Kac-Moody algebras. Let $I$ be an index set. A square matrix $\mathrm{A}=\left(a_{i j}\right)_{i, j \in I}$ is called a symmetrizable Borcherds-Cartan matrix if it satisfies (i) $a_{i i}=2$ or $a_{i i} \in 2 \mathbb{Z}_{\leq 0}$ for $i \in I$, (ii) $a_{i j} \in \mathbb{Z}_{\leq 0}$ for $i \neq j$, (iii) $a_{i j}=0$ if $a_{j i}=0$ for $i, j \in I$, (vi) there is a diagonal matrix $\mathrm{D}=\operatorname{diag}\left(\mathrm{d}_{i} \in \mathbb{Z}_{>0} \mid i \in I\right)$ such that DA is symmetric. Let $I^{\mathrm{re}}=\left\{i \in I \mid a_{i i}=2\right\}$ and $I^{\mathrm{im}}=I \backslash I^{\mathrm{re}}$.

A symmetrizable Borcherds-Cartan datum (A, $\left.\mathrm{P}, \Pi, \Pi^{\vee}\right)$ consists of

(1) a symmetrizable Borcherds-Cartan matrix A, 
(2) a free abelian group $\mathrm{P}$, called the weight lattice,

(3) the set $\Pi=\left\{\alpha_{i} \mid i \in I\right\} \subset \mathrm{P}$ of simple roots,

(4) the set $\Pi^{\vee}=\left\{h_{i} \mid i \in I\right\} \subset \mathrm{P}^{\vee}:=\operatorname{Hom}(\mathrm{P}, \mathbb{Z})$ of simple coroots,

which satisfy the following properties:

(i) $\left\langle h_{i}, \alpha_{j}\right\rangle:=\alpha_{j}\left(h_{i}\right)=a_{i j}$ for all $i, j \in I$,

(ii) $\Pi \subset \mathfrak{h}^{*}$ is linearly independent, where $\mathfrak{h}:=\mathbb{C} \otimes_{\mathbb{Z}} \mathrm{P}^{\vee}$,

(iii) for each $i \in I$, there exists $\Lambda_{i} \in \mathrm{P}$ such that $\left\langle h_{j}, \Lambda_{i}\right\rangle=\delta_{i j}$ for all $j \in I$.

We denote by $\mathrm{P}^{+}=\left\{\lambda \in \mathrm{P} \mid \lambda\left(h_{i}\right) \in \mathbb{Z}_{\geq 0}, i \in I\right\}$ the set of dominant integral weights. The free abelian group $\mathrm{Q}=\bigoplus_{i \in I} \mathbb{Z} \alpha_{i}$ is the root lattice, and $\mathrm{Q}^{+}=\sum_{i \in I} \mathbb{Z}_{\geq 0} \alpha_{i}$ is the positive root lattice. For $\alpha=\sum_{i \in I} k_{i} \alpha_{i} \in \mathrm{Q}^{+}$, the height $|\alpha|$ of $\alpha$ is $\sum_{i \in I} k_{i}$. There is a symmetric bilinear form $(\mid)$ on $\mathfrak{h}^{*}$ such that

$$
\left(\alpha_{i} \mid \lambda\right)=\mathrm{d}_{i}\left\langle h_{i}, \lambda\right\rangle \text { for } \lambda \in \mathfrak{h}^{*}, i \in I .
$$

Thus we have $\left(\alpha_{i} \mid \alpha_{j}\right)=\mathrm{d}_{i} a_{i j}$ for $i, j \in I$.

Let $q$ be an indeterminate and $m, n \in \mathbb{Z}_{\geq 0}$. For $i \in I^{\mathrm{re}}$, let $q_{i}=q^{\mathrm{d}_{i}}$ and

$$
[n]_{i}=\frac{q_{i}^{n}-q_{i}^{-n}}{q_{i}-q_{i}^{-1}}, \quad[n]_{i} !=\prod_{k=1}^{n}[k]_{i}, \quad\left[\begin{array}{c}
m \\
n
\end{array}\right]_{i}=\frac{[m]_{i} !}{[m-n]_{i} ![n]_{i} !} .
$$

Definition 1.1. The quantum generalized Kac-Moody algebra $U_{q}(\mathfrak{g})$ associated with a BorcherdsCartan datum $\left(\mathrm{A}, \mathrm{P}, \Pi, \Pi^{\vee}\right)$ is the associative algebra over $\mathbb{C}(q)$ with 1 generated by $e_{i}, f_{i}$ $(i \in I)$ and $q^{h}\left(h \in \mathrm{P}^{\vee}\right)$ satisfying following relations:

(1) $q^{0}=1, q^{h} q^{h^{\prime}}=q^{h+h^{\prime}}$ for $h, h^{\prime} \in \mathrm{P}^{\vee}$,

(2) $q^{h} e_{i} q^{-h}=q^{\left\langle h, \alpha_{i}\right\rangle} e_{i}, q^{h} f_{i} q^{-h}=q^{-\left\langle h, \alpha_{i}\right\rangle} f_{i}$ for $h \in \mathrm{P}^{\vee}, i \in I$,

(3) $e_{i} f_{j}-f_{j} e_{i}=\delta_{i j} \frac{K_{i}-K_{i}^{-1}}{q_{i}-q_{i}^{-1}}$, where $K_{i}=q_{i}^{h_{i}}$,

(4) $\sum_{k=0}^{1-a_{i j}}(-1)^{k}\left[\begin{array}{c}1-a_{i j} \\ k\end{array}\right]_{i} e_{i}^{1-a_{i j}-k} e_{j} e_{i}^{k}=0$ if $i \in I^{\mathrm{re}}$ and $i \neq j$,

(5) $\sum_{k=0}^{1-a_{i j}}(-1)^{k}\left[\begin{array}{c}1-a_{i j} \\ k\end{array}\right]_{i} f_{i}^{1-a_{i j}-k} f_{j} f_{i}^{k}=0$ if $i \in I^{\mathrm{re}}$ and $i \neq j$,

(6) $e_{i} e_{j}-e_{j} e_{i}=0, f_{i} f_{j}-f_{j} f_{i}=0$ if $a_{i j}=0$.

We denote by $U_{q}^{-}(\mathfrak{g})$ the subalgebra of $U_{q}(\mathfrak{g})$ generated by the elements $f_{i}(i \in I)$. Let us set $\mathbb{A}=\mathbb{Z}\left[q, q^{-1}\right]$ and we denote by $U_{\mathbb{A}}^{-}(\mathfrak{g})$ the $\mathbb{A}$-subalgebra of $U_{q}(\mathfrak{g})$ generated by $f_{i}^{(n)}$ $\left(i \in I^{\mathrm{re}}\right.$ and $\left.n \in \mathbb{Z}_{>0}\right)$ and $f_{i}\left(i \in I^{\mathrm{im}}\right)$.

For a dominant integral weight $\lambda \in \mathrm{P}^{+}$, let $V(\lambda)$ be the irreducible highest weight $U_{q}(\mathfrak{g})$ module with highest weight $\lambda$ and let $V_{\mathbb{A}}(\lambda)$ denote the $U_{\mathbb{A}}^{-}(\mathfrak{g})$-submodule of $V(\lambda)$ generated by the highest weight vector. 
1.2. Khovanov-Lauda-Rouquier algebras. For $\alpha \in \mathrm{Q}^{+}$with $|\alpha|=m$, let $I^{\alpha}=\{\nu=$ $\left.\left(\nu_{1}, \ldots, \nu_{m}\right) \in I^{m} \mid \alpha_{\nu_{1}}+\cdots+\alpha_{\nu_{m}}=\alpha\right\}$. The symmetric group $\mathrm{S}_{m}=\left\langle s_{k} \mid k=1, \ldots, m-1\right\rangle$ acts naturally on $I^{\alpha}$; i.e., for $w \in \mathrm{S}_{m}$ and $\nu=\left(\nu_{1}, \ldots, \nu_{m}\right) \in I^{\alpha}$,

$$
w \nu=\left(\nu_{w^{-1}(1)}, \ldots, \nu_{w^{-1}(m)}\right)
$$

Let $\mathbf{k}=\bigoplus_{n \in \mathbb{Z}} \mathbf{k}_{n}$ be a commutative graded ring such that $\mathbf{k}_{n}=0$ for $n<0$. The symmetric group $\mathrm{S}_{m}$ acts on the polynomial ring $\mathbf{k}\left[x_{1}, \ldots, x_{m}\right]$ by

$$
w\left(f\left(x_{1}, \ldots, x_{m}\right)\right)=f\left(x_{w(1)}, \ldots, x_{w(m)}\right) \quad \text { for } w \in \mathrm{S}_{m} \text { and } f\left(x_{1}, \ldots, x_{m}\right) \in \mathbf{k}\left[x_{1}, \ldots, x_{m}\right]
$$

For $t=1, \ldots, m-1$, define the operator $\partial_{t}$ on $\mathbf{k}\left[x_{1}, \ldots, x_{m}\right]$ by

$$
\partial_{t}(f)=\frac{s_{t} f-f}{x_{t}-x_{t+1}}
$$

for $f \in \mathbf{k}\left[x_{1}, \ldots, x_{m}\right]$.

We take a matrix $\left(\mathcal{Q}_{i, j}(u, v)\right)_{i, j \in I}$ in $\mathbf{k}[u, v]$ such that $\mathcal{Q}_{i, j}(u, v)=\mathcal{Q}_{j, i}(v, u)$ and $\mathcal{Q}_{i, j}(u, v)$ has the form

$$
\mathcal{Q}_{i, j}(u, v)= \begin{cases}\sum_{p, q \geq 0} t_{i, j ; p, q} u^{p} v^{q} & \text { if } i \neq j, \\ 0 & \text { if } i=j\end{cases}
$$

where $t_{i, j ; p, q} \in \mathbf{k}_{-2\left(\alpha_{i} \mid \alpha_{j}\right)-2 \mathrm{~d}_{i} p-2 \mathrm{~d}_{j} q}$ and $t_{i, j ;-a_{i j}, 0} \in \mathbf{k}_{0}^{\times}$. For each $i \in I$, we choose a polynomial $\mathcal{P}_{i}(u, v) \in \mathbf{k}[u, v]$ having the form

$$
\mathcal{P}_{i}(u, v)=\sum_{p, q \geq 0} h_{i ; p, q} u^{p} v^{q}
$$

where $h_{i ; p, q} \in \mathbf{k}_{\mathrm{d}_{i}\left(2-a_{i i}\right)-2 \mathrm{~d}_{i} p-2 \mathrm{~d}_{i} q}$ and $h_{i ; 1-\frac{a_{i i}}{2}, 0}, h_{i ; 0,1-\frac{a_{i i}}{2}} \in \mathbf{k}_{0}^{\times}$.

Definition $1.2([9,10])$. Let $\left(\mathrm{A}, \mathrm{P}, \Pi, \Pi^{\vee}\right)$ be a Borcherds-Cartan datum. For $\alpha \in \mathrm{Q}^{+}$ with height $m$, the Khovanov-Lauda-Rouquier algebra $R(\alpha)$ of weight $\alpha$ associated with the data $\left(\mathrm{A}, \mathrm{P}, \Pi, \Pi^{\vee}\right),\left(\mathcal{P}_{i}\right)_{i \in I}$ and $\left(\mathcal{Q}_{i, j}\right)_{i, j \in I}$ is the associative graded $\mathbf{k}$-algebra generated by $\mathrm{e}(\nu)\left(\nu \in I^{\alpha}\right), \mathrm{x}_{k}(1 \leq k \leq m)$ and $\mathrm{r}_{t}(1 \leq t \leq m-1)$ satisfying the following defining relations: 


$$
\begin{aligned}
& \mathrm{e}(\nu) \mathrm{e}\left(\nu^{\prime}\right)=\delta_{\nu, \nu^{\prime}} \mathrm{e}(\nu), \sum_{\nu \in I^{\alpha}} \mathrm{e}(\nu)=1, \mathrm{x}_{k} \mathrm{e}(\nu)=\mathrm{e}(\nu) \mathrm{x}_{k}, \mathrm{x}_{k} \mathrm{x}_{l}=\mathrm{x}_{l} \mathrm{x}_{k}, \\
& \mathrm{r}_{t} \mathrm{e}(\nu)=\mathrm{e}\left(s_{t}(\nu)\right) \mathrm{r}_{t}, \mathrm{r}_{t} \mathrm{r}_{s}=\mathrm{r}_{s} \mathrm{r}_{t} \text { if }|t-s|>1, \\
& \mathrm{r}_{t}^{2} \mathrm{e}(\nu)= \begin{cases}\partial_{t} \mathcal{P}_{\nu_{t}}\left(\mathrm{x}_{t}, \mathrm{x}_{t+1}\right) \mathrm{r}_{t} \mathrm{e}(\nu) & \text { if } \nu_{t}=\nu_{t+1}, \\
\mathcal{Q}_{\nu_{t}, \nu_{t+1}}\left(\mathrm{x}_{t}, \mathrm{x}_{t+1}\right) \mathrm{e}(\nu) & \text { if } \nu_{t} \neq \nu_{t+1},\end{cases} \\
& \left(\mathrm{r}_{t} \mathrm{x}_{k}-\mathrm{x}_{s t}(k) \mathrm{r}_{t}\right) \mathrm{e}(\nu)= \begin{cases}-\mathcal{P}_{\nu_{t}}\left(\mathrm{x}_{t}, \mathrm{x}_{t+1}\right) \mathrm{e}(\nu) & \text { if } k=t \text { and } \nu_{t}=\nu_{t+1}, \\
\mathcal{P}_{\nu_{t}}\left(\mathrm{x}_{t}, \mathrm{x}_{t+1}\right) \mathrm{e}(\nu) & \text { if } k=t+1 \text { and } \nu_{t}=\nu_{t+1}, \\
0 & \text { otherwise, }\end{cases} \\
& \left(\mathrm{r}_{t+1} \mathrm{r}_{t} \mathrm{r}_{t+1}-\mathrm{r}_{t} \mathrm{r}_{t+1} \mathrm{r}_{t}\right) \mathrm{e}(\nu) \\
& = \begin{cases}\mathcal{P}_{\nu_{t}}\left(\mathrm{x}_{t}, \mathrm{x}_{t+2}\right) \overline{\mathcal{Q}}_{\nu_{t}, \nu_{t+1}}\left(\mathrm{x}_{t}, \mathrm{x}_{t+1}, \mathrm{x}_{t+2}\right) \mathrm{e}(\nu) \\
\overline{\mathcal{P}}_{\nu_{t}}^{\prime}\left(\mathrm{x}_{t}, \mathrm{x}_{t+1}, \mathrm{x}_{t+2}\right) \mathrm{r}_{t} \mathrm{e}(\nu)+\overline{\mathcal{P}}_{\nu_{t}}^{\prime \prime}\left(\mathrm{x}_{t}, \mathrm{x}_{t+1}, \mathrm{x}_{t+2}\right) \mathrm{r}_{t+1} \mathrm{e}(\nu) & \text { if } \nu_{t}=\nu_{t+1}=\nu_{t+2}, \\
0 & \text { otherwise, },\end{cases}
\end{aligned}
$$

where

$$
\begin{aligned}
\overline{\mathcal{P}}_{i}^{\prime}(u, v, w) & :=\frac{\mathcal{P}_{i}(v, u) \mathcal{P}_{i}(u, w)}{(u-v)(u-w)}+\frac{\mathcal{P}_{i}(u, w) \mathcal{P}_{i}(v, w)}{(u-w)(v-w)}-\frac{\mathcal{P}_{i}(u, v) \mathcal{P}_{i}(v, w)}{(u-v)(v-w)}, \\
\overline{\mathcal{P}}_{i}^{\prime \prime}(u, v, w) & :=-\frac{\mathcal{P}_{i}(u, v) \mathcal{P}_{i}(u, w)}{(u-v)(u-w)}-\frac{\mathcal{P}_{i}(u, w) \mathcal{P}_{i}(w, v)}{(u-w)(v-w)}+\frac{\mathcal{P}_{i}(u, v) \mathcal{P}_{i}(v, w)}{(u-v)(v-w)}, \\
\overline{\mathcal{Q}}_{i, j}(u, v, w) & :=\frac{\mathcal{Q}_{i, j}(u, v)-\mathcal{Q}_{i, j}(w, v)}{u-w} .
\end{aligned}
$$

For $\nu \in I^{\alpha}$, set $\mathrm{x}_{k}(\nu)=\mathrm{x}_{k} \mathrm{e}(\nu)$ and $\mathrm{r}_{t}(\nu)=\mathrm{r}_{t} \mathrm{e}(\nu)$. Then the $\mathbb{Z}$-grading on $R(\alpha)$ is given by

$$
\operatorname{deg}(\mathrm{e}(\nu))=0, \quad \operatorname{deg}\left(\mathrm{x}_{k}(\nu)\right)=2 \mathrm{~d}_{\nu_{k}}, \quad \operatorname{deg}\left(\mathrm{r}_{t}(\nu)\right)=-\left(\alpha_{\nu_{t}} \mid \alpha_{\nu_{t+1}}\right) .
$$

For $\lambda \in \mathrm{P}^{+}$and $i \in I$, let us choose a polynomial $a_{i}^{\lambda}(u)$ of the from

$$
a_{i}^{\lambda}(u)=\sum_{k=0}^{\lambda\left(h_{i}\right)} c_{i ; k}^{\lambda} u^{\lambda\left(h_{i}\right)-k}
$$

where $c_{i ; k}^{\lambda} \in \mathbf{k}_{2 \mathrm{~d}_{i} k}$ and $c_{i ; 0}^{\lambda}=1$. We set $a^{\lambda}(\mathrm{x})=\sum_{\nu \in I^{\alpha}} a_{\nu_{1}}^{\lambda}\left(\mathrm{x}_{1}\right) \mathrm{e}(\nu)$. Then the cyclotomic Khovanov-Lauda-Rouquier algebra $R^{\lambda}(\alpha)$ at $\alpha$ is defined to be the quotient algebra

$$
R^{\lambda}(\alpha)=R(\alpha) / R(\alpha) a^{\lambda}(\mathrm{x}) R(\alpha) .
$$

Assume that $\mathbf{k}_{0}$ is a field. Let

$$
K_{0}(R)=\bigoplus_{\alpha \in Q^{+}} K_{0}(R(\alpha) \text {-pmod }), \quad K_{0}\left(R^{\lambda}\right)=\bigoplus_{\alpha \in Q^{+}} K_{0}\left(R^{\lambda}(\alpha) \text {-pmod }\right),
$$


where $K_{0}\left(R(\alpha)\right.$-pmod) (resp. $K_{0}\left(R^{\lambda}(\alpha)\right.$-pmod)) is the Grothendieck group of the category $R(\alpha)$-pmod (resp. $R^{\lambda}(\alpha)$-pmod) of finitely generated projective graded left $R(\alpha)$-modules (resp. finitely generated projective graded left $R^{\lambda}(\alpha)$-modules). Then $K_{0}(R)\left(\operatorname{resp} . K_{0}\left(R^{\lambda}\right)\right.$ ) has the $\mathbb{A}$-module structure induced by the $\mathbb{Z}$-grading on $R\left(\operatorname{resp} . R^{\lambda}\right)$, where $\mathbb{A}:=\mathbb{Z}\left[q, q^{-1}\right]$.

For $\beta, \beta^{\prime} \in \mathrm{Q}^{+}$, we define

$$
\mathrm{e}\left(\beta, \beta^{\prime}\right)=\sum_{\nu \in I^{\beta}, \nu^{\prime} \in I^{\beta^{\prime}}} \mathrm{e}\left(\nu, \nu^{\prime}\right) \in R\left(\beta+\beta^{\prime}\right)
$$

where $\mathrm{e}\left(\nu, \nu^{\prime}\right)$ is the idempotent corresponding to the concatenation of $\nu$ and $\nu^{\prime}$. For each $i \in I$ and $\alpha, \beta \in \mathrm{Q}^{+}$, we define the functors

$$
\begin{array}{ll}
\operatorname{Res}_{\alpha, \beta} & : R(\alpha+\beta) \text {-pmod } \longrightarrow R(\alpha) \otimes R(\beta) \text {-pmod, } \\
\operatorname{Ind}_{\alpha, \beta} & : R(\alpha) \otimes R(\beta) \text {-pmod } \longrightarrow R(\alpha+\beta) \text {-pmod }
\end{array}
$$

by

$$
\begin{aligned}
& \operatorname{Res}_{\alpha, \beta}(N)=\mathrm{e}(\alpha, \beta) N, \\
& \operatorname{Ind}_{\alpha, \beta}(L)=R(\alpha+\beta) \mathrm{e}(\alpha, \beta) \underset{R(\alpha) \otimes R(\beta)}{\otimes} L
\end{aligned}
$$

for $L \in R(\alpha) \otimes R(\beta)$-pmod and $N \in R(\alpha+\beta)$-pmod. One can show that $K_{0}(R(\alpha)$-pmod $) \otimes_{\mathbb{A}}$ $K_{0}(R(\beta)$-pmod $) \rightarrow K_{0}\left(R(\alpha) \otimes R(\beta)\right.$-pmod) is an isomorphism, and $K_{0}(R)$ becomes a bialgebra $[10,13]$.

For a $\mathbb{Z}$-graded module $M=\bigoplus_{k \in \mathbb{Z}} M_{k}$ and $t \in \mathbb{Z}$, let $M\langle t\rangle$ be the $\mathbb{Z}$-graded module defined by $M\langle t\rangle_{k}=M_{k+t}$. For each $i \in I^{\text {re }}$ and $m \geq 0$, we define the projective graded $R\left(m \alpha_{i}\right)$-module $P\left(i^{m}\right)$ to be

$$
P\left(i^{m}\right)=\frac{R\left(m \alpha_{i}\right)}{\sum_{t=1}^{m-1} R\left(m \alpha_{i}\right) r_{t}}\left\langle\frac{m(m-1)\left(\alpha_{i} \mid \alpha_{i}\right)}{4}\right\rangle .
$$

If $i \in I^{\mathrm{im}}$, we define $P(i)=R\left(\alpha_{i}\right)$, the regular representation. The following theorem was proved in [10] (see also [13]).

Theorem $1.3([10])$. (i) There exists an injective bialgebra homomorphism $\Phi: U_{\mathbb{A}}^{-}(\mathfrak{g}) \longrightarrow$ $K_{0}(R)$ sending $f_{i}^{(m)}$ to $P\left(i^{m}\right)\left(i \in I^{\mathrm{re}}\right)$ and $f_{i}$ to $P(i)\left(i \in I^{\mathrm{im}}\right)$.

(ii) If $a_{i i} \neq 0$ for any $i \in I$, then $\Phi$ is an isomorphism.

For a dominant integral weight $\lambda \in \mathrm{P}^{+}$, we define the functors

$$
\begin{aligned}
& E_{i}^{\lambda}: R^{\lambda}\left(\beta+\alpha_{i}\right) \text {-pmod } \longrightarrow R^{\lambda}(\beta) \text {-pmod }, \\
& F_{i}^{\lambda}: R^{\lambda}(\beta)-\operatorname{pmod} \longrightarrow R^{\lambda}\left(\beta+\alpha_{i}\right) \text {-pmod }
\end{aligned}
$$


by

$$
E_{i}^{\lambda}(N)=\mathrm{e}\left(\beta, \alpha_{i}\right) N, \quad F_{i}^{\lambda}(M)=R^{\lambda}\left(\beta+\alpha_{i}\right) \mathrm{e}\left(\beta, \alpha_{i}\right) \otimes_{R^{\lambda}(\beta)} M
$$

for $M \in R^{\lambda}(\beta)$-pmod and $N \in R^{\lambda}\left(\beta+\alpha_{i}\right)$-pmod, respectively. Then the functors $E_{i}^{\lambda}, F_{i}^{\lambda}$ define a $U_{\mathbb{A}}(\mathfrak{g})$-module structure on $K_{0}\left(R^{\lambda}\right)$. Let $V_{\mathbb{A}}(\lambda)$ be the $\mathbb{A}$-form of the irreducible highest weight $U_{q}(\mathfrak{g})$-module $V(\lambda)$. The following generalized version of cyclotomic categorification conjecture was proved in $[9]$.

Theorem $1.4([8,9])$. If $a_{i i} \neq 0$ for any $i \in I$, then there exists a $U_{\mathbb{A}}(\mathfrak{g})$-module isomorphism $\Phi^{\lambda}: V_{\mathbb{A}}(\lambda) \stackrel{\sim}{\longrightarrow} K_{0}\left(R^{\lambda}\right)$.

In this paper, in order to give a geometric realization of $R(\alpha)$, we assume that $\mathrm{A}$ is symmetric.

Then, we can take $\mathrm{d}_{i}=1$ and $\left(\alpha_{i} \mid \alpha_{j}\right)=a_{i j}$ for $i \neq j \in I$. Set $\mathrm{I}_{i}=1-a_{i i} / 2$ for $i \in I$ and choose non-negative integers $\mathrm{h}_{i j}$ such that $a_{i j}=-\mathrm{h}_{i j}-\mathrm{h}_{j i}$ for $i \neq j \in I$. Let $\Lambda_{i, j}(i, j \in I)$ be an index set of $\mathrm{h}_{i, j}$ elements for $i \neq j$ and of $\mathrm{I}_{i}$ elements for $i=j$. We set $\Lambda=\bigsqcup_{i, j \in I} \Lambda_{i, j}$. Let $\mathrm{H}$ be the polynomial ring over $\mathbb{C}$ generated by indeterminates $\hbar_{a}(a \in \Lambda)$ with $\operatorname{deg}\left(\hbar_{a}\right)=2$; i.e.,

$$
\mathrm{H} \simeq \bigotimes_{a \in \Lambda} \mathbb{C}\left[\hbar_{a}\right]
$$

We take $\mathbf{H}$ as the base ring $\mathbf{k}$ and consider the specially chosen polynomials $\mathcal{P}_{i}(u, v)$ and $\mathcal{Q}_{i, j}(u, v)$ in $\mathrm{H}[u, v]$ given as follows:

$$
\begin{aligned}
\mathcal{P}_{i}(u, v) & =\prod_{a \in \Lambda_{i, i}}\left(u-v+\hbar_{a}\right), \\
\mathcal{Q}_{i, j}(u, v) & = \begin{cases}0 & \text { if } i=j, \\
\prod_{a \in \Lambda_{i, j}}\left(v-u+\hbar_{a}\right) \prod_{a \in \Lambda_{j, i}}\left(u-v+\hbar_{a}\right) & \text { if } i \neq j .\end{cases}
\end{aligned}
$$

In this case, the algebras $R(\alpha)$ has another $\mathbb{Z}$-grading given as follows:

$$
\operatorname{deg}(\mathrm{e}(\nu))=0, \quad \operatorname{deg}\left(\mathrm{x}_{k}(\nu)\right)=2, \quad \operatorname{deg}\left(\mathrm{r}_{l}(\nu)\right)= \begin{cases}2\left(\mathrm{I}_{\nu_{l}}-1\right) & \text { if } \nu_{l}=\nu_{l+1} \\ 2 \mathrm{~h}_{\nu_{l}, \nu_{l+1}} & \text { if } \nu_{l} \neq \nu_{l+1}\end{cases}
$$

We now construct a faithful graded polynomial representation of $R(\alpha)$ associated with $\mathrm{A}$, (1.2.6) and (1.2.7). Set

$$
\operatorname{Pol}(\alpha)=\bigoplus_{\nu \in I^{\alpha}} \mathrm{H}\left[\mathrm{x}_{1}(\nu), \ldots, \mathrm{x}_{m}(\nu)\right]
$$

with $\operatorname{deg}\left(\mathrm{x}_{k}(\nu)\right)=2$. For $f \in \mathrm{H}\left[x_{1}, \ldots, x_{m}\right]$, we denote by $f(\nu)$ the element $f\left(\mathrm{x}_{1}(\nu), \ldots, \mathrm{x}_{m}(\nu)\right) \in$ $\mathrm{H}\left[\mathrm{x}_{1}(\nu), \ldots, \mathrm{x}_{m}(\nu)\right]$. 
Then the following proposition is proved in [10, 13, 18].

Proposition 1.5. The algebra $\operatorname{Pol}(\alpha)$ has a graded $R(\alpha)$-module structure as follows: for $\nu=\left(\nu_{1}, \ldots, \nu_{m}\right), \nu^{\prime} \in I^{\alpha}$ and $f \in \mathrm{H}\left[x_{1}, \ldots, x_{m}\right]$,

$$
\begin{aligned}
\mathrm{e}(\nu) \cdot f\left(\nu^{\prime}\right) & =\delta_{\nu, \nu^{\prime}} f(\nu), \\
\mathrm{x}_{k}(\nu) \cdot f\left(\nu^{\prime}\right) & =\delta_{\nu, \nu^{\prime}} \mathrm{x}_{k}(\nu) f(\nu), \\
\mathrm{r}_{l}(\nu) \cdot f\left(\nu^{\prime}\right) & = \begin{cases}0 & \text { if } \nu \neq \nu^{\prime}, \\
\left(\prod_{a \in \Lambda_{\nu_{l}, \nu_{l+1}}}\left(\mathrm{x}_{l}\left(s_{l} \nu\right)-\mathrm{x}_{l+1}\left(s_{l} \nu\right)+\hbar_{a}\right)\right)\left(s_{l} f\right)\left(s_{l} \nu\right) & \text { if } \nu=\nu^{\prime}, s_{l} \nu \neq \nu, \\
\left(\prod_{a \in \Lambda_{\nu_{l}, \nu_{l}}}\left(\mathrm{x}_{l}(\nu)-\mathrm{x}_{l+1}(\nu)+\hbar_{a}\right)\right)\left(\partial_{l} f\right)(\nu) & \text { if } \nu=\nu^{\prime}, s_{l} \nu=\nu .\end{cases}
\end{aligned}
$$

Moreover, $\operatorname{Pol}(\alpha)$ is a faithful $R(\alpha)$-module.

\section{Geometric realization}

In this section, we give a geometric realization of $R(\alpha)$. We first construct the Steinbergtype variety $\mathcal{Z}_{\nu, \nu^{\prime}}$ out of a given locally finite quiver. We then investigate the convolution algebra $\mathfrak{R}(\alpha)=\bigoplus_{\nu, \nu^{\prime} \in I^{\alpha}} H_{*}^{\mathrm{G}_{\alpha}^{\Omega}}\left(\mathcal{Z}_{\nu, \nu^{\prime}}\right)\left\langle-2 \operatorname{dim}_{\mathbb{C}} \widetilde{\mathcal{F}}_{\nu}\right\rangle$ arising from $\mathcal{Z}_{\nu, \nu^{\prime}}$ and show that the algebra $\mathfrak{R}(\alpha)$ is isomorphic to the Khovanov-Lauda-Rouquier algebra $R(\alpha)$ using the faithful representations $\operatorname{Pol}(\alpha)$ and $\mathfrak{P o l}(\alpha)$ given in Proposition 1.5 and Proposition 2.7.

2.1. Quiver representation varieties. Let $Q=(I, \Omega)$ be an arbitrary locally finite quiver with a vertex set $I$ and an oriented edge set $\Omega$. For $a \in \Omega$, let in $(a)(\operatorname{resp}$. out $(a))$ denote the incoming vertex (resp. the outgoing vertex) of $a$. For $i, j, k \in I$ with $j \neq k$, let

$$
\begin{aligned}
& \Omega_{i, j}=\{a \in \Omega \mid \operatorname{out}(a)=i, \operatorname{in}(a)=j\}, \\
& \ell_{i}=\# \Omega_{i, i} \text { and } h_{j, k}=\# \Omega_{j, k} .
\end{aligned}
$$

Let $U_{q}(\mathfrak{g})$ be the quantum generalized Kac-Moody algebra associated with the BorcherdsCartan matrix $\mathrm{A}=\left(a_{i j}\right)_{i, j \in I}$ defined by

$$
a_{i i}=2-2 \ell_{i}, \quad a_{j k}=-h_{j k}-h_{k j}
$$

for $i, j, k \in I$ with $j \neq k$. Note that $I^{\text {re }}=\left\{i \in I \mid \ell_{i}=0\right\}, I^{\text {im }}=\left\{i \in I \mid \ell_{i} \neq 0\right\}$.

Define the torus $\mathrm{H}^{\Omega}$ corresponding to the oriented edge set $\Omega$ by

$$
\mathrm{H}^{\Omega}=\prod_{a \in \Omega} \mathbb{C}^{*}
$$

and denote its Lie algebra by $\mathfrak{h}^{\Omega}$. 
Let $\alpha \in \mathrm{Q}^{+}$and let $m=|\alpha|$. Fix an $I$-graded $\mathbb{C}$-vector space $V_{\alpha}=\bigoplus_{i \in I} V_{i}$ with $\underline{\operatorname{dim}} V_{\alpha}:=$ $\sum_{i \in I}\left(\operatorname{dim} V_{i}\right) \alpha_{i}=\alpha$. Let $\mathrm{G}_{i}=G L\left(V_{i}\right)(i \in I)$ and $\mathrm{G}_{\alpha}=\prod_{i \in I} G L\left(V_{i}\right)$. We denote by $\mathfrak{g}_{\alpha}=\bigoplus_{i \in I} \mathfrak{g l}\left(V_{i}\right)$ the Lie algebra of $\mathrm{G}_{\alpha}$ and identify $\mathfrak{g}_{\alpha}$ with its dual $\mathfrak{g}_{\alpha}^{*}$ via $\sum_{i \in I} \operatorname{tr}_{V_{i}}$. Let

$$
\mathrm{G}_{\alpha}^{\Omega}=\mathrm{G}_{\alpha} \times \mathrm{H}^{\Omega}
$$

We define the quiver representation variety to be

$$
\mathcal{E}_{\alpha}=\bigoplus_{a \in \Omega} \operatorname{Hom}\left(V_{\mathrm{out}(a)}, V_{\operatorname{in}(a)}\right) .
$$

The group $G_{\alpha}^{\Omega}$ acts on $\mathcal{E}_{\alpha}$ by

$$
(g, t) \cdot x=\left(t_{a}\left(g_{\operatorname{in}(a)} x_{a} g_{\text {out }(a)}^{-1}\right)\right)_{a \in \Omega}
$$

for $x=\left(x_{a}\right)_{a \in \Omega} \in \mathcal{E}_{\alpha}$ and $(g, t)=\left(\left(g_{i}\right)_{i \in I},\left(t_{a}\right)_{a \in \Omega}\right) \in \mathrm{G}_{\alpha}^{\Omega}$.

For $\nu=\left(\nu_{1}, \ldots, \nu_{m}\right) \in I^{\alpha}$, let

$$
\begin{aligned}
\mathcal{F}_{\nu}=\{F=(0= & \left.F_{0} \subset F_{1} \subset \cdots \subset F_{m}=V_{\alpha}\right) \\
& \left.\mid F_{k} \text { is } I \text {-graded and } \underline{\operatorname{dim}}\left(F_{k} / F_{k-1}\right)=\alpha_{\nu_{k}} \text { for } k=1, \ldots, m\right\}
\end{aligned}
$$

be the variety of complete flags of type $\nu$ and set $\mathcal{F}_{\alpha}=\bigsqcup_{\nu \in I^{\alpha}} \mathcal{F}_{\nu}$. For a vector space $W$, we denote by $\mathcal{F}(W)$ the variety consisting of complete flags of $W$. Then there is an isomorphism

$$
\mathcal{F}_{\nu} \stackrel{\sim}{\longrightarrow} \prod_{i \in I} \mathcal{F}\left(V_{i}\right), \quad F \mapsto\left({ }^{i} E\right)_{i \in I},
$$

where ${ }^{i} E_{k}=V_{i} \cap F_{k}$ for $k=1, \ldots, m$ and ${ }^{i} E=\left(0={ }^{i} E_{0} \subset{ }^{i} E_{1} \subset \cdots \subset{ }^{i} E_{m}=V_{i}\right) \in \mathcal{F}\left(V_{i}\right)$ for $i \in I$. The group $\mathrm{G}_{\alpha}$ acts transitively on $\mathcal{F}_{\nu}$ and $\prod_{i \in I} \mathcal{F}\left(V_{i}\right)$, and $\mathrm{H}^{\Omega}$ acts trivially on them, so that the isomorphism (2.1.2) is $\mathrm{G}_{\alpha}^{\Omega}$-equivariant.

For $x \in \mathcal{E}_{\alpha}$ and $F=\left(0=F_{0} \subset F_{1} \subset \cdots \subset F_{m}\right) \in \mathcal{F}_{\nu}$ is said to be strictly $x$-stable if $x_{a}\left(F_{k} \cap V_{\text {out }(a)}\right) \subset F_{k-1} \cap V_{\operatorname{in}(a)}$ for all $a \in \Omega$ and $k=1, \ldots, m$. Let

$$
\widetilde{\mathcal{F}}_{\nu}=\left\{(x, F) \in \mathcal{E}_{\alpha} \times \mathcal{F}_{\nu} \mid F \text { is strictly } x \text {-stable }\right\}
$$

and let $\widetilde{\mathcal{F}}_{\alpha}=\bigsqcup_{\nu \in I^{\alpha}} \widetilde{\mathcal{F}}_{\nu}$. Then $G_{\alpha}^{\Omega}$ acts diagonally on $\widetilde{\mathcal{F}}_{\nu}$ and $\widetilde{\mathcal{F}}_{\nu}$ is a $G_{\alpha}^{\Omega}$-equivariant vector bundle over $\mathcal{F}_{\nu}$.

Let us consider the following maps

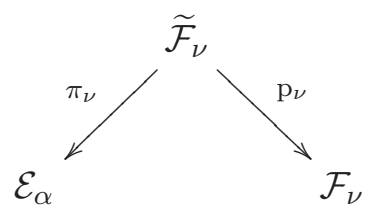


defined by $\pi_{\nu}(x, F)=x$ and $\mathrm{p}_{\nu}(x, F)=F$ for $(x, F) \in \widetilde{\mathcal{F}}_{\nu}$. Both of the maps $\pi_{\nu}$ and $\mathrm{p}_{\nu}$ are $\mathrm{G}_{\alpha}^{\Omega}$-equivariant and $\pi_{\nu}$ is projective. For $\nu, \nu^{\prime} \in I^{\alpha}$, we define the Steinberg-type variety

$$
\begin{aligned}
\mathcal{Z}_{\nu, \nu^{\prime}} & =\widetilde{\mathcal{F}}_{\nu} \times \mathcal{E}_{\alpha} \widetilde{\mathcal{F}}_{\nu^{\prime}} \\
& \simeq\left\{\left(x, F, F^{\prime}\right) \in \mathcal{E}_{\alpha} \times \mathcal{F}_{\nu} \times \mathcal{F}_{\nu^{\prime}} \mid(x, F) \in \widetilde{\mathcal{F}}_{\nu},\left(x, F^{\prime}\right) \in \widetilde{\mathcal{F}}_{\nu^{\prime}}\right\},
\end{aligned}
$$

and let $\mathcal{Z}_{\alpha}=\bigsqcup_{\nu, \nu^{\prime} \in I^{\alpha}} \mathcal{Z}_{\nu, \nu^{\prime}}$. Consider the map

$$
\mathrm{q}_{\nu, \nu^{\prime}}: \mathcal{Z}_{\nu, \nu^{\prime}} \longrightarrow \mathcal{F}_{\nu} \times \mathcal{F}_{\nu^{\prime}}
$$

defined by $\mathrm{q}_{\nu, \nu^{\prime}}\left(x, F, F^{\prime}\right)=\left(F, F^{\prime}\right)$ for $\left(x, F, F^{\prime}\right) \in \mathcal{Z}_{\nu, \nu^{\prime}}$, and set

$$
\mathrm{q}_{\alpha}:=\bigsqcup_{\nu, \nu^{\prime} \in I^{\alpha}} \mathrm{q}_{\nu, \nu^{\prime}}: \mathcal{Z}_{\alpha} \longrightarrow \mathcal{F}_{\alpha} \times \mathcal{F}_{\alpha} .
$$

Then the map $\mathrm{q}_{\nu, \nu^{\prime}}$ is a $\mathrm{G}_{\alpha}^{\Omega}$-equivariant morphism.

From now on, we fix $\nu^{\circ}=\left(\nu_{1}^{\circ}, \ldots, \nu_{m}^{\circ}\right) \in I^{\alpha}$ and $F^{\circ} \in \mathcal{F}_{\nu^{\circ}}$. Set $\mathrm{G}=G L\left(V_{\alpha}\right)$ and let $\mathrm{B}$ be the stabilizer of $F^{\circ}$ in $\mathrm{G}$. Then $\mathrm{B}$ and $\mathrm{B}_{\alpha}:=\mathrm{B} \cap \mathrm{G}_{\alpha}$ are Borel subgroups of $\mathrm{G}$ and $\mathrm{G}_{\alpha}$, respectively. Take a maximal torus $\mathrm{T}$ of $\mathrm{B}_{\alpha}$ and let

$$
\mathrm{T}^{\Omega}=\mathrm{T} \times \mathrm{H}^{\Omega} .
$$

We denote by $\mathfrak{g}\left(\right.$ resp. $\left.\mathfrak{b}, \mathfrak{b}_{\alpha}, \mathfrak{t}\right)$ the Lie algebra of $\mathrm{G}$ (resp. B, $\mathrm{B}_{\alpha}, \mathrm{T}$ ).

There exist 1-dimensional T-submodules $\mathbb{F}_{1}, \ldots, \mathbb{F}_{m}$ of $V_{\alpha}$ such that

$$
F^{\circ}=\left(0 \subset \mathbb{F}_{1} \subset \mathbb{F}_{1} \oplus \mathbb{F}_{2} \subset \cdots \subset \mathbb{F}_{1} \oplus \cdots \oplus \mathbb{F}_{m}=V_{\alpha}\right) .
$$

Note that $\mathbb{F}_{k} \subset V_{\nu_{k}^{\circ}}$ for $k=1, \ldots, m$. For $w, w^{\prime} \in \mathrm{S}_{m}$, set $\nu_{w}=\left(\nu_{w(1)}^{\circ}, \ldots, \nu_{w(m)}^{\circ}\right)$ and

$$
\begin{aligned}
& F_{w}=\left(0 \subset \mathbb{F}_{w(1)} \subset \mathbb{F}_{w(1)} \oplus \mathbb{F}_{w(2)} \subset \cdots \subset \mathbb{F}_{w(1)} \oplus \cdots \oplus \mathbb{F}_{w(m)}=V_{\alpha}\right) \in \mathcal{F}_{\nu_{w}}, \\
& F_{w, w^{\prime}}=\left(F_{w}, F_{w^{\prime}}\right) \in \mathcal{F}_{\nu_{w}} \times \mathcal{F}_{\nu_{w^{\prime}}} .
\end{aligned}
$$

Let $\mathrm{B}_{\alpha, w}$ be the stabilizer of $F_{w}$ in $\mathrm{G}_{\alpha}$. Let $\mathrm{W}$ and $\mathrm{W}_{\alpha}$ be the Weyl group of the pair $(\mathrm{G}, \mathrm{T})$ and $\left(\mathrm{G}_{\alpha}, \mathrm{T}\right)$, respectively. We identify $\mathrm{W}$ with the symmetric group $\mathrm{S}_{m}$ via $w \cdot \mathbb{F}_{k}=\mathbb{F}_{w(k)}$ for $w \in \mathrm{W}$ and $k=1, \ldots, m$; i.e., $w \cdot F^{\circ}=F_{w}$. Note that $w \nu_{w^{\prime}}=\nu_{w^{\prime} w^{-1}}$ for $w, w^{\prime} \in \mathrm{W}$ by (1.2.1). The quotient set $\mathrm{W}_{\alpha} \backslash \mathrm{W}$ is in 1-1 correspondence with $I^{\alpha}$ via the map sending $w$ to $\nu_{w}$.

For $w \in \mathrm{W}$, set

$$
\mathfrak{e}_{w}=\left\{x \in \mathcal{E}_{\alpha} \mid F_{w} \text { is strictly } x \text {-stable }\right\} \simeq \mathrm{p}_{\nu_{w}}^{-1}\left(F_{w}\right) .
$$

By the definition, we have

$$
\begin{aligned}
\mathfrak{e}_{w} & =\left\{x \in \mathcal{E}_{\alpha} \mid x\left(\mathbb{F}_{w(k)}\right) \subset \mathbb{F}_{w(1)} \oplus \cdots \oplus \mathbb{F}_{w(k-1)} \text { for } 1 \leq k \leq m\right\} \\
& \simeq \bigoplus_{m \geq k>k^{\prime} \geq 1} \operatorname{Hom}\left(\mathbb{F}_{w(k)}, \mathbb{F}_{w\left(k^{\prime}\right)}\right)^{\oplus \Omega_{(\nu w)},(\nu w)_{k^{\prime}} .}
\end{aligned}
$$


Similarly, the Lie algebra $\mathfrak{b}_{\alpha, w}$ of $B_{\alpha, w}$ is calculated as

$$
\begin{aligned}
\mathfrak{b}_{\alpha, w} & \left.=\left\{x \in \bigoplus_{i \in I} \operatorname{End}\left(V_{i}\right) \mid x\left(\mathbb{F}_{w(k)}\right) \subset \mathbb{F}_{w(1)} \oplus \cdots \oplus \mathbb{F}_{w(k)} \text { for } 1 \leq k \leq m\right)\right\} \\
& \simeq \bigoplus_{\substack{m \geq k \geq k^{\prime} \geq 1,\left(\nu_{w}\right)_{k}=\left(\nu_{w}\right)_{k^{\prime}}}} \operatorname{Hom}\left(\mathbb{F}_{w(k)}, \mathbb{F}_{w\left(k^{\prime}\right)}\right) .
\end{aligned}
$$

For $w \in \mathrm{W}$ and $\nu, \nu^{\prime} \in I^{\alpha}$, we define

$$
\begin{aligned}
\mathcal{O}_{\alpha}^{w} & =\mathrm{G} \cdot F_{e, w} \cap\left(\mathcal{F}_{\alpha} \times \mathcal{F}_{\alpha}\right), & \overline{\mathcal{O}}_{\alpha}^{w} & =\text { the closure of } \mathcal{O}_{\alpha}^{w} \text { in } \mathcal{F}_{\alpha} \times \mathcal{F}_{\alpha}, \\
\mathcal{O}_{\nu, \nu^{\prime}}^{w} & =\mathcal{O}_{\alpha}^{w} \cap\left(\mathcal{F}_{\nu} \times \mathcal{F}_{\nu^{\prime}}\right), & \overline{\mathcal{O}}_{\nu, \nu^{\prime}}^{w} & =\overline{\mathcal{O}}_{\alpha}^{w} \cap\left(\mathcal{F}_{\nu} \times \mathcal{F}_{\nu^{\prime}}\right) .
\end{aligned}
$$

Note that we have a Bruhat decomposition $\mathcal{F}_{\alpha} \times \mathcal{F}_{\alpha}=\bigsqcup_{w \in \mathrm{W}} \mathcal{O}_{\alpha}^{w}$. For $w, w^{\prime}, w^{\prime \prime} \in \mathrm{W}$, we abbreviate

$$
\mathcal{O}_{w^{\prime}, w^{\prime \prime}}^{w}=\mathcal{O}_{\nu_{w^{\prime}}, \nu_{w^{\prime \prime}}}^{w}, \quad \overline{\mathcal{O}}_{w^{\prime}, w^{\prime \prime}}^{w}=\overline{\mathcal{O}}_{\nu_{w^{\prime}}, \nu_{w^{\prime \prime}}}^{w} .
$$

Lemma 2.1. Let $w \in \mathrm{W}, \nu, \nu^{\prime} \in I^{\alpha}$ and let $s_{i} \in \mathrm{W}$ be a simple reflection.

(i) The set of elements of $\mathcal{O}_{\alpha}^{w}$ fixed under the diagonal action of $\mathrm{T}$ is $\left\{F_{u, u w} \mid u \in \mathrm{W}\right\}$.

(ii) If $\nu^{\prime} \neq w^{-1} \nu$, then $\overline{\mathcal{O}}_{\nu, \nu^{\prime}}^{w}=\emptyset$.

(iii) We have

$$
\overline{\mathcal{O}}_{\alpha}^{s_{i}}=\bigsqcup_{\nu \in I^{\alpha}}\left\{\left(F, F^{\prime}\right) \in \mathcal{F}_{\nu} \times \mathcal{F}_{s_{i} \nu} \mid F_{k}=F_{k}^{\prime} \quad \text { for any } k \neq i\right\} .
$$

In particular, $\overline{\mathcal{O}}_{\nu, s_{i} \nu}^{s_{i}}$ is smooth and $\operatorname{dim}_{\mathbb{C}} \overline{\mathcal{O}}_{\nu, s_{i} \nu}^{s_{i}}=\operatorname{dim}_{\mathbb{C}} \mathcal{F}_{\nu}+\delta_{\nu, s_{i} \nu}$.

(iv) If $\nu=s_{i} \nu$, then $\overline{\mathcal{O}}_{\nu, \nu}^{s_{i}}=\mathcal{O}_{\nu, \nu}^{s_{i}} \cup \mathcal{O}_{\nu, \nu}^{e}$, and the first and the second projections $\overline{\mathcal{O}}_{\nu, \nu}^{s_{i}} \rightarrow \mathcal{F}_{\nu}$ are $\mathbb{P}^{1}$-bundles.

(v) If $\nu \neq s_{i} \nu$, then $\overline{\mathcal{O}}_{\nu, s_{i} \nu}^{s_{i}}=\mathcal{O}_{\nu, s_{i} \nu}^{s_{i}}$ and $\overline{\mathcal{O}}_{\nu, \nu}^{s_{i}}=\emptyset$. Moreover the projections $\overline{\mathcal{O}}_{\nu, s_{i} \nu}^{s_{i}} \rightarrow \mathcal{F}_{\nu}$ and $\overline{\mathcal{O}}_{\nu, s_{i} \nu}^{s_{i}} \rightarrow \mathcal{F}_{s_{i} \nu}$ are isomorphisms.

(vi) If $w^{-1} \nu=\nu^{\prime}$, then $\mathcal{O}_{\nu, \nu^{\prime}}^{w}$ is a $\mathrm{G}_{\alpha}$-orbit and it is an affine fibration over $\mathcal{F}_{\nu}$ with fiber being isomorphic to the affine space $\mathrm{B}_{\alpha, u} \cdot F_{u w}$ for $u \in W$ such that $\nu=\nu_{u}$.

Proof. We shall only prove (ii) and (vi), since the other assertions are easily proved (see [20, Lemma 2.6]). Since $\mathcal{O}_{\nu, \nu^{\prime}}^{w}$ is $\mathrm{G}_{\alpha}$-invariant, $\mathcal{O}_{\nu, \nu^{\prime}}^{w}$ is a union of $G_{\alpha}$-orbits, and any $\mathrm{G}_{\alpha}$-orbit of $\mathcal{F}_{\alpha} \times \mathcal{F}_{\alpha}$ contains a T-fixed point.

We first show the assertion (ii). Suppose that $w^{-1} \nu \neq \nu^{\prime}$. If $\overline{\mathcal{O}}_{\nu, \nu^{\prime}}^{w}$ is not empty, then $\mathcal{O}_{\nu, \nu^{\prime}}^{w}$ contains a T-fixed point $F_{u, u w}$ for some $u \in W$. Then we have $\nu=\nu_{u}$ and $\nu^{\prime}=\nu_{u w}=w^{-1} \nu_{u}$, which is a contradiction.

Now consider the case $w^{-1} \nu=\nu^{\prime}$. Choose a T-fixed point $F_{u, u w} \in \mathcal{O}_{\nu, \nu^{\prime}}^{w}$ for some $u \in W$. Then $\nu=\nu_{u}$ and $\nu^{\prime}=\nu_{u w}$. If we take another T-fixed point $F_{u^{\prime}, u^{\prime} w} \in \mathcal{O}_{\nu, \nu^{\prime}}^{w}$, then there is an element $v \in \mathrm{W}_{\alpha}$ such that

$$
v \cdot F_{u}=F_{v u}=F_{u^{\prime}}
$$


which yields $F_{u^{\prime}, u^{\prime} w}=v \cdot F_{u, u w} \in \mathrm{G}_{\alpha} \cdot F_{u, u w}$. Therefore,

$$
\mathcal{O}_{\nu, \nu^{\prime}}^{w}=\mathrm{G}_{\alpha} \cdot F_{u, u w}
$$

Let $\phi_{\nu, \nu^{\prime}}^{w}: \mathcal{O}_{\nu, \nu^{\prime}}^{w} \rightarrow \mathcal{F}_{\nu}$ be the map defined by $\phi_{\nu, \nu^{\prime}}^{w}\left(F, F^{\prime}\right)=F$ for $\left(F, F^{\prime}\right) \in \mathcal{O}_{\nu, \nu^{\prime}}^{w}$. Then $\phi_{\nu, \nu^{\prime}}^{w}$ is surjective since $\phi_{\nu, \nu^{\prime}}^{w}$ is $\mathrm{G}_{\alpha^{-}}$-equivariant, and the fiber of $g \cdot F_{u}$ is given as follows:

$$
\begin{aligned}
\phi_{\nu, \nu^{\prime}}^{w}{ }^{-1}\left(g \cdot F_{u}\right) & =g \cdot\left\{\left(F_{u}, F^{\prime}\right) \mid F^{\prime} \in \mathrm{B}_{\alpha, u} \cdot F_{u w}\right\} \\
& \simeq \mathrm{B}_{\alpha, u} \cdot F_{u w} .
\end{aligned}
$$

Since $\mathrm{B}_{\alpha, u} \cdot F_{u w}$ is an affine space [4], we obtain (vi).

2.2. The convolution algebra. For a given quasi-projective variety $X$ over $\mathbb{C}$ with an action of a complex linear algebraic group $G$, let $\mathbf{D}_{G}^{b}(X)$ be the bounded $G$-equivariant derived category of sheaves of $\mathbb{C}$-vector spaces on $X$. Let $\mathbb{C}_{X}$ be the constant sheaf on $X$ and let $\varpi_{X} \in \mathbf{D}_{G}^{b}(X)$ be the $G$-equivariant dualizing complex. For any complex $\mathcal{L} \in \mathbf{D}_{G}^{b}(X)$, let $H_{G}^{k}(X, \mathcal{L})=\operatorname{Hom}_{\mathbf{D}_{\mathrm{G}}^{b}(X)}\left(\mathbb{C}_{X}, \mathcal{L}[k]\right)$, where [.] is the translation functor. For $k \in \mathbb{Z}$, define

$$
H_{k}^{G}(X)=H_{G}^{k}\left(X, \varpi_{X}\right), \quad H_{*}^{G}(X)=\bigoplus_{k \in \mathbb{Z}} H_{k}^{G}(X)
$$

and

$$
H_{G}^{k}(X)=H_{G}^{k}\left(X, \mathbb{C}_{X}\right), \quad H_{G}^{*}(X)=\bigoplus_{k \in \mathbb{Z}} H_{G}^{k}(X) .
$$

Note that, if $X \hookrightarrow M$ is a closed embedding into a smooth variety $M$, then we have $H_{k}^{G}(X)=$ $H_{G}^{k+2 \operatorname{dim}_{\mathbb{C}} M}(M, M \backslash X)$. The graded module $H_{G}^{*}(X)$ has a graded ring structure and $H_{*}^{G}(X)$ has a graded $H_{G}^{*}(X)$-module structure. We refer to $[2,3,5]$ for more details on equivariant cohomologies.

We now return to our setting. Let $\mathbf{H}$ be the ring given by

$$
\mathbf{H}=H_{\mathrm{H}^{\Omega}}^{*}(\mathrm{pt}) \simeq \bigotimes_{a \in \Omega} \mathbb{C}\left[\hbar_{a}\right]
$$

where $\hbar_{a} \in H_{\mathrm{H}^{\Omega}}^{*}(\mathrm{pt})$ corresponds to the one-dimensional representation of the $a$-th factor $\mathbb{C}^{*}$ of $\mathrm{H}^{\Omega}$. This will play the role of the base ring given in (1.2.5). Let $\mathbf{S}$ be the ring defined by

$$
\mathbf{S}=H_{\mathrm{G}_{\alpha}^{\Omega}}^{*}(\mathrm{pt}) \simeq \mathbf{H} \otimes H_{\mathrm{G}_{\alpha}}^{*}(\mathrm{pt})
$$

For $\nu, \nu^{\prime} \in I^{\alpha}$, we define

$$
\begin{array}{ll}
\mathfrak{R}_{\nu, \nu^{\prime}}:=H_{*}^{\mathrm{G}_{\alpha}^{\Omega}}\left(\mathcal{Z}_{\nu, \nu^{\prime}}\right)\left\langle-2 \operatorname{dim}_{\mathbb{C}} \widetilde{\mathcal{F}}_{\nu}\right\rangle, & \mathfrak{R}(\alpha):=\bigoplus_{\nu, \nu^{\prime} \in I^{\alpha}} \mathfrak{R}_{\nu, \nu^{\prime}}, \\
\mathfrak{P o l}_{\nu}:=H_{\mathrm{G}_{\alpha}^{\Omega}}^{*}\left(\widetilde{\mathcal{F}}_{\nu}\right), & \mathfrak{P o l}(\alpha):=H_{\mathrm{G}_{\alpha}^{\Omega}}^{*}\left(\widetilde{\mathcal{F}}_{\alpha}\right) \simeq \bigoplus_{\nu \in I^{\alpha}} \mathfrak{P o l}_{\nu} .
\end{array}
$$


For $k=1, \ldots, m$, let $\chi_{k} \in \mathfrak{t}^{*}$ be the weight of the $\mathfrak{t}$-module induced from the T-module $\mathbb{F}_{k}$ given in (2.1.6) by differentiation. Then we have

$$
H_{\mathrm{T}^{\Omega}}^{*}(\mathrm{pt}) \simeq \mathbf{H} \otimes H_{\mathrm{T}}^{*}(\mathrm{pt}) \simeq \mathbf{H}\left[\chi_{1}, \ldots, \chi_{m}\right] .
$$

For $\nu \in I^{\alpha}$, we denote by $\mathcal{L}_{\nu, k}$ the $G_{\alpha}^{\Omega}$-equivariant line bundle on $\mathcal{F}_{\nu}$ assigning $F_{k} / F_{k-1}$ to a flag $0=F_{0} \subset F_{1} \subset \cdots \subset F_{m}=V$ in $\mathcal{F}_{\nu}$. Let $\chi_{k}(\nu)$ be the $\mathrm{G}_{\alpha}^{\Omega}$-equivariant first Chern class $c_{1}\left(\mathrm{p}_{\nu}^{*} \mathcal{L}_{\nu, k}\right) \in H_{\mathrm{G}_{\alpha}^{\Omega}}^{2}\left(\widetilde{\mathcal{F}}_{\nu}\right)$. For any $w \in \mathrm{W}$ such that $\nu=\nu_{w}$, we have isomorphisms

$$
H_{\mathrm{G}_{\alpha}^{\Omega}}^{*}\left(\widetilde{\mathcal{F}}_{\nu}\right) \simeq H_{\mathrm{G}_{\alpha}^{\Omega}}^{*}\left(\mathcal{F}_{\nu}\right) \simeq H_{\mathrm{G}_{\alpha}^{\Omega}}^{*}\left(\mathrm{G}_{\alpha}^{\Omega} /\left(\mathrm{B}_{\alpha, w} \times \mathrm{H}^{\Omega}\right)\right) \simeq H_{\mathrm{T}^{\Omega}}^{*}(\mathrm{pt})
$$

Moreover we see that

$$
\left\{\begin{array}{l}
\text { the composition of these isomorphisms coincides with the composition } \\
\qquad H_{\mathrm{G}_{\alpha}^{\Omega}}^{*}\left(\widetilde{\mathcal{F}}_{\nu}\right) \rightarrow H_{\mathrm{T}^{\Omega}}^{*}\left(\widetilde{\mathcal{F}}_{\nu}\right) \rightarrow H_{\mathrm{T}^{\Omega}}^{*}\left(\left\{\left(0, F_{w}\right)\right\}\right) \simeq H_{\mathrm{T}^{\Omega}}^{*}(\mathrm{pt}) \simeq \mathbf{H}\left[\chi_{1}, \ldots, \chi_{m}\right] \\
\text { and it sends } \chi_{k}(\nu) \text { to } \chi_{w(k)} .
\end{array}\right.
$$

The last assertion follows from the fact that the stalk of $\mathrm{p}_{\nu}^{*} \mathcal{L}_{\nu, k}$ at $\left(0, F_{w}\right) \in \widetilde{\mathcal{F}}_{\nu}$ is isomorphic to $\mathbb{F}_{w(k)}$ as a $\mathrm{T}^{\Omega}$-module.

Therefore, we have

$$
\mathfrak{P o l}_{\nu}=H_{\mathrm{G}_{\alpha}^{\Omega}}^{*}\left(\widetilde{\mathcal{F}}_{\nu}\right) \simeq \mathbf{H}\left[\chi_{1}(\nu), \ldots, \chi_{m}(\nu)\right]
$$

as an algebra, and

$$
\mathfrak{P o r}(\alpha)=\bigoplus_{\nu \in I^{\alpha}} H_{\mathrm{G}_{\alpha}^{\Omega}}^{*}\left(\widetilde{\mathcal{F}}_{\nu}\right) \simeq \bigoplus_{\nu \in I^{\alpha}} \mathbf{H}\left[\chi_{1}(\nu), \ldots, \chi_{m}(\nu)\right]
$$

For $\nu, \nu^{\prime}, \nu^{\prime \prime} \in I^{\alpha}$, let $\mathfrak{p}_{\nu, \nu^{\prime}}$ (resp. $\mathfrak{p}_{\nu^{\prime}, \nu^{\prime \prime}}, \mathfrak{p}_{\nu, \nu^{\prime \prime}}$ ) be the natural projection from $\widetilde{\mathcal{F}}_{\nu} \times \widetilde{\mathcal{F}}_{\nu^{\prime}} \times \widetilde{\mathcal{F}}_{\nu^{\prime \prime}}$ to $\widetilde{\mathcal{F}}_{\nu} \times \widetilde{\mathcal{F}}_{\nu^{\prime}}\left(\right.$ resp. $\left.\widetilde{\mathcal{F}}_{\nu^{\prime}} \times \widetilde{\mathcal{F}}_{\nu^{\prime \prime}}, \widetilde{\mathcal{F}}_{\nu} \times \widetilde{\mathcal{F}}_{\nu^{\prime \prime}}\right)$. Since $\mathfrak{p}_{\nu, \nu^{\prime}}^{-1}\left(\mathcal{Z}_{\nu, \nu^{\prime}}\right) \cap \mathfrak{p}_{\nu^{\prime}, \nu^{\prime \prime}}^{-1}\left(\mathcal{Z}_{\nu^{\prime}, \nu^{\prime \prime}}\right) \simeq \widetilde{\mathcal{F}}_{\nu} \times \mathcal{E}_{\alpha} \widetilde{\mathcal{F}}_{\nu^{\prime}} \times \mathcal{E}_{\alpha} \widetilde{\mathcal{F}}_{\nu^{\prime \prime}}$, the restriction of the natural projection

$$
\mathfrak{p}_{\nu, \nu^{\prime \prime}}: \mathfrak{p}_{\nu, \nu^{\prime}}^{-1}\left(\mathcal{Z}_{\nu, \nu^{\prime}}\right) \cap \mathfrak{p}_{\nu^{\prime}, \nu^{\prime \prime}}^{-1}\left(\mathcal{Z}_{\nu^{\prime}, \nu^{\prime \prime}}\right) \longrightarrow \mathcal{Z}_{\nu, \nu^{\prime \prime}}
$$

is proper. Hence, for $c_{1} \in H_{p}^{\mathrm{G}_{\alpha}^{\Omega}}\left(\mathcal{Z}_{\nu, \nu^{\prime}}\right)$ and $c_{2} \in H_{q}^{\mathrm{G}_{\alpha}^{\Omega}}\left(\mathcal{Z}_{\nu^{\prime}, \nu^{\prime \prime}}\right)$, the convolution product

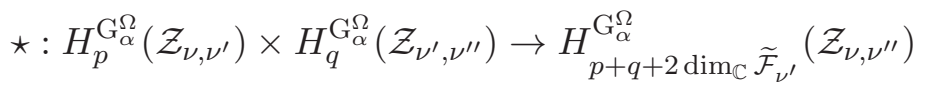

is defined by

$$
c_{1} \star c_{2}=\left(\mathfrak{p}_{\nu, \nu^{\prime \prime}}\right)_{*}\left(\mathfrak{p}_{\nu, \nu^{\prime}}^{*}\left(c_{1}\right) \cap \mathfrak{p}_{\nu^{\prime}, \nu^{\prime \prime}}^{*}\left(c_{2}\right)\right),
$$

where $\cap$ is the intersection pairing [5]. Then $\mathfrak{R}(\alpha)$ becomes an associative graded $\mathbf{H}$-algebra with the convolution product $\star$. Moreover $\mathfrak{P o l}(\alpha)$ has the graded $\mathfrak{R}(\alpha)$-module structure 
arising from the convolution product:

$$
\begin{aligned}
& H_{p}^{\mathrm{G}_{\alpha}^{\Omega}}\left(\mathcal{Z}_{\nu, \nu^{\prime}}\right) \otimes H_{\mathrm{G}_{\alpha}^{\Omega}}^{q}\left(\widetilde{\mathcal{F}}_{\nu^{\prime}}\right) \rightarrow H_{p}^{\mathrm{G}_{\alpha}^{\Omega}}\left(\mathcal{Z}_{\nu, \nu^{\prime}}\right) \otimes H_{\mathrm{G}_{\alpha}^{\Omega}}^{q}\left(\mathcal{Z}_{\nu, \nu^{\prime}}\right) \\
& \rightarrow \quad H_{p+q}^{\mathrm{G}_{\alpha}^{\Omega}}\left(\mathcal{Z}_{\nu, \nu^{\prime}}\right) \rightarrow H_{p+q}^{\mathrm{G}_{\alpha}^{\Omega}}\left(\widetilde{\mathcal{F}}_{\nu}\right) \simeq H_{\mathrm{G}_{\alpha}^{\Omega}}^{p+q+2 \operatorname{dim}_{\mathbb{C}}} \widetilde{\mathcal{F}}_{\nu}\left(\widetilde{\mathcal{F}}_{\nu}\right) .
\end{aligned}
$$

Here, the first arrow is the pull-back by the projection $\mathcal{Z}_{\nu, \nu^{\prime}} \rightarrow \widetilde{\mathcal{F}}_{\nu^{\prime}}$, the second arrow is the multiplication and the last arrow is the push-forward by the proper map $\mathcal{Z}_{\nu, \nu^{\prime}} \rightarrow \widetilde{\mathcal{F}}_{\nu}$.

Recall the maps $\mathrm{q}_{\nu, \nu^{\prime}}: \mathcal{Z}_{\nu, \nu^{\prime}} \rightarrow \mathcal{F}_{\nu} \times \mathcal{F}_{\nu^{\prime}}$ and $\mathrm{q}_{\alpha}: \mathcal{Z}_{\alpha} \rightarrow \mathcal{F}_{\alpha} \times \mathcal{F}_{\alpha}$ given in (2.1.4) and (2.1.5). For $w \in \mathrm{W}$ and $\nu, \nu^{\prime} \in I^{\alpha}$, we define

$$
\begin{array}{ll}
\mathcal{Z}_{\alpha}^{w}=\overline{\mathrm{q}_{\alpha}^{-1}\left(\mathcal{O}_{\alpha}^{w}\right)}, & \mathcal{Z}_{\alpha}^{\leq w}=\bigcup_{w^{\prime} \leq w} \mathcal{Z}_{\alpha}^{w^{\prime}}, \\
\mathcal{Z}_{\nu, \nu^{\prime}}^{w}=\mathcal{Z}_{\alpha}^{w} \cap \mathcal{Z}_{\nu, \nu^{\prime}}, & \mathcal{Z}_{\nu, \nu^{\prime}}^{\leq w}=\mathcal{Z}_{\alpha}^{\leq w} \cap \mathcal{Z}_{\nu, \nu^{\prime}},
\end{array}
$$

where $\overline{\mathrm{q}_{\alpha}^{-1}\left(\mathcal{O}_{\alpha}^{w}\right)}$ is the closure in $\mathcal{Z}_{\alpha}$. Note that $\mathcal{Z}_{\alpha}^{\leq w}=\bigcup_{w^{\prime} \leq w} \mathrm{q}_{\alpha}^{-1}\left(\mathcal{O}_{\alpha}^{w^{\prime}}\right)$ is a closed $\mathrm{G}_{\alpha}^{\Omega}$ subvariety.

Lemma 2.2. For $\nu, \nu^{\prime} \in I^{\alpha} w \in \mathrm{W}$ and $j=1, \ldots, m-1$, we have

(i) If $\nu^{\prime} \neq w^{-1} \nu$, then $\mathcal{Z}_{\nu, \nu^{\prime}}^{w}=\emptyset$. If $\nu^{\prime}=w^{-1} \nu$, then $\mathcal{Z}_{\nu, \nu^{\prime}}^{w}$ is an irreducible variety.

(ii) $\mathcal{Z}_{\alpha}^{s_{j}}=\left\{\left(x, F, F^{\prime}\right) \in \mathcal{E}_{\alpha} \times \mathcal{F}_{\alpha} \times \mathcal{F}_{\alpha}\right.$

$$
\left.\mid F_{k}=F_{k}^{\prime} \text { for } k \neq j, x F_{k} \subset F_{k-1} \text { for } k \neq j, j+1 \text { and } x F_{j+1} \subset F_{j-1}\right\} .
$$

(iii) $\mathcal{Z}_{\alpha}^{s_{j}}$ is a smooth variety.

Proof. (i) follows immediately from Lemma 2.1 (ii) and the fact that $\mathrm{q}_{\alpha}^{-1}\left(\mathcal{O}_{\alpha}^{w}\right) \rightarrow \mathcal{O}_{\alpha}^{w}$ is a vector bundle.

Let us show (ii). The right-hand side is a vector bundle over $\overline{\mathcal{O}}_{\alpha}^{s_{j}}$ by Lemma 2.1 (iii). Since $F_{j} \cap F_{j}^{\prime}=F_{j-1}$ for $\left(F, F^{\prime}\right) \in \mathcal{O}_{\alpha}^{s_{j}}, \mathrm{q}_{\alpha}^{-1} \mathcal{O}_{\alpha}^{s_{j}}$ is an open dense subset of the right-hand side of (ii).

(iii) follows immediately from (ii).

For $w \in \mathrm{W}$ and $\nu, \nu^{\prime} \in I^{\alpha}$, let

$$
\mathfrak{R}_{\nu, \nu^{\prime}}^{\leq w}=H_{*}^{\mathrm{G}_{\alpha}^{\Omega}}\left(\mathcal{Z}_{\nu, \nu^{\prime}}^{\leq w}\right)\left\langle-2 \operatorname{dim}_{\mathbb{C}} \widetilde{\mathcal{F}}_{\nu}\right\rangle, \quad \mathfrak{R}_{\alpha}^{\leq w}=\bigoplus_{\nu, \nu^{\prime} \in I^{\alpha}} \mathfrak{R}_{\nu, \nu^{\prime}}^{\leq w}
$$

For $w \in \mathrm{W}$, we denote by $\left[\mathcal{Z}_{\alpha}^{w}\right]=\sum_{\nu, \nu^{\prime}}\left[\mathcal{Z}_{\nu, \nu^{\prime}}^{w}\right]$ the class in $\mathfrak{R} \leq w$ arising from $\mathcal{Z}_{\alpha}^{w}=\bigsqcup_{\nu, \nu^{\prime}} \mathcal{Z}_{\nu, \nu^{\prime}}^{w}$. The convolution $\star$ gives a left $\mathfrak{R}_{\alpha}^{\leq e}$-module structure on the space $\mathfrak{R} \frac{\leq w}{\alpha}$. Using $\mathcal{Z}_{\bar{\nu}, \nu}^{\leq e} \simeq \widetilde{\mathcal{F}}_{\nu}$, the isomorphism (2.2.5) yields

$$
H_{*}^{\mathrm{G}_{\alpha}^{\Omega}}\left(\mathcal{Z}_{\nu, \nu}^{\leq e}\right)\left\langle-2 \operatorname{dim}_{\mathbb{C}} \widetilde{\mathcal{F}}_{\nu}\right\rangle \simeq H_{\mathrm{G}_{\alpha}^{\Omega}}^{*}\left(\mathcal{Z}_{\nu, \nu}^{\leq e}\right) \simeq H_{\mathrm{G}_{\alpha}^{\Omega}}^{*}\left(\widetilde{\mathcal{F}}_{\nu}\right) \simeq \mathbf{H}\left[\chi_{1}(\nu), \ldots, \chi_{m}(\nu)\right] .
$$


Let $\varkappa_{k}(\nu) \in H_{\mathrm{G}_{\alpha}^{\Omega}}^{*}\left(\mathcal{Z}_{\nu, \nu}^{\leq e}\right)$ be the image of $\chi_{k}(\nu)$ under the isomorphism $(2.2 .9)$ for $k=1, \ldots, m$. Then we have

$$
\mathfrak{R}_{\alpha}^{\leq e} \simeq \bigoplus_{\nu \in I^{\alpha}} H_{\mathrm{G}_{\alpha}^{\Omega}}^{*}\left(\mathcal{Z}_{\nu, \nu}^{\leq e}\right) \simeq \bigoplus_{\nu \in I^{\alpha}} \mathbf{H}\left[\varkappa_{1}(\nu), \ldots, \varkappa_{m}(\nu)\right]
$$

as an algebra.

The following lemma will play an important role in proving our main result.

\section{Lemma 2.3.}

(i) The closed embedding $\mathcal{Z}_{\alpha}^{\leq w} \hookrightarrow \mathcal{Z}_{\alpha}$ induces an injective graded $\mathfrak{R} \leq e$-bimodule homomorphism $\mathfrak{R} \leq w \hookrightarrow \mathfrak{R}(\alpha)$.

(ii) For $w \in \mathrm{W}, \mathfrak{R} \leq w$ is a free $\mathfrak{R}_{\alpha}^{\leq e}$-module of rank $\#\left\{w^{\prime} \in \mathrm{W} \mid w^{\prime} \leq w\right\}$ and

$$
\mathfrak{R} \leq w=\bigoplus_{w^{\prime} \leq w} \mathfrak{R}_{\alpha}^{\leq e} \star\left[\mathcal{Z}_{\alpha}^{w^{\prime}}\right] .
$$

(iii) If $\ell\left(s_{j} w\right)=\ell(w)+1$, we have

$$
\left[\mathcal{Z}_{\alpha}^{s_{j}}\right] \star\left[\mathcal{Z}_{\alpha}^{w}\right]=\left[\mathcal{Z}_{\alpha}^{s_{j} w}\right] \quad \text { in } \mathfrak{R}_{\alpha}^{\leq s_{j} w} / \mathfrak{R}_{\alpha}^{<s_{j} w},
$$

where $\mathfrak{R}_{\alpha}^{<u}:=\sum_{u^{\prime}<u} \mathfrak{R} \leq u^{\prime}$ for $u \in \mathrm{W}$.

Proof. Since (i) is a consequence of (ii), we first prove (ii). Let $\ell=\#\left\{w^{\prime} \in \mathrm{W} \mid w^{\prime} \leq w\right\}$. We give a total order $\prec$ on $\left\{w^{\prime} \in \mathrm{W} \mid w^{\prime} \leq w\right\}$ by

$$
e=w_{1} \prec \cdots \prec w_{k} \prec w_{k+1} \prec \cdots \prec w_{\ell}=w
$$

such that $\ell\left(w_{k+1}\right)=\ell\left(w_{k}\right)$ or $\ell\left(w_{k+1}\right)=\ell\left(w_{k}\right)+1$ for all $k$. Set

$$
\mathcal{Z}^{\preceq k}=\bigcup_{i \leq k} \mathcal{Z}_{\alpha}^{w_{i}} \text { for } k=1, \ldots, \ell .
$$

Then $\mathcal{Z}^{\preceq k}$ is a closed subset of $\mathcal{Z}_{\alpha}$. Since $\mathcal{Z}_{\alpha}^{\leq w}=\mathcal{Z}^{\preceq \ell}$, it suffices to show that

$$
H_{*}^{\mathrm{G}_{\alpha}^{\Omega}}\left(\mathcal{Z}^{\preceq k}\right)=\bigoplus_{k^{\prime} \leq k} \mathfrak{R}_{\alpha}^{\leq e} \star\left[\mathcal{Z}_{\alpha}^{w_{k^{\prime}}}\right], \quad H_{2 t+1}^{\mathrm{G}_{\alpha}^{\Omega}}\left(\mathcal{Z}^{\preceq k}\right)=0 \quad \text { for all } k=1, \ldots, \ell .
$$

We use induction on $k$. Assume $k=1$. Since $\operatorname{deg}\left(\varkappa_{k}(\nu)\right)=2$ for $k=1, \ldots, m$ and $\nu \in I^{\alpha}$, (2.2.10) implies

$$
H_{*}^{\mathrm{G}_{\alpha}^{\Omega}}\left(\mathcal{Z}^{\preceq 1}\right)=\mathfrak{R}_{\alpha}^{\leq e} \star\left[\mathcal{Z}_{\alpha}^{e}\right], \quad H_{2 t+1}^{\mathrm{G}_{\alpha}^{\Omega}}\left(\mathcal{Z}^{\preceq 1}\right)=0 \quad \text { for } t \in \mathbb{Z} .
$$

Suppose $k>1$. By the induction hypothesis, we have

$$
H_{*}^{\mathrm{G}_{\alpha}^{\Omega}}\left(\mathcal{Z}^{\preceq k-1}\right)=\bigoplus_{k^{\prime} \leq k-1} \Re_{\alpha}^{\leq e} \star\left[\mathcal{Z}_{\alpha}^{w_{k^{\prime}}}\right], \quad H_{2 t+1}^{\mathrm{G}_{\alpha}^{\Omega}}\left(\mathcal{Z}^{\preceq k-1}\right)=0 \quad \text { for } t \in \mathbb{Z} .
$$


Let $\mathrm{q}_{k}=\left.\mathrm{q}_{\alpha}\right|_{\mathcal{Z} \preceq k}: \mathcal{Z} \preceq k \rightarrow \mathcal{F}_{\alpha} \times \mathcal{F}_{\alpha}$ and consider the following diagram:

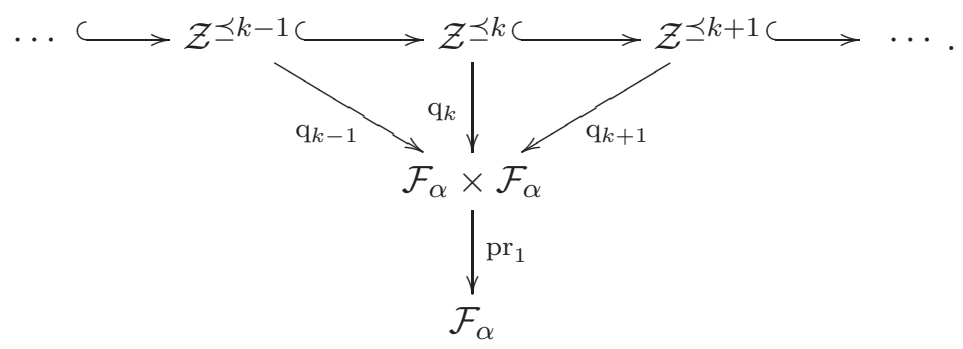

Here, $\mathrm{pr}_{1}: \mathcal{F}_{\alpha} \times \mathcal{F}_{\alpha} \rightarrow \mathcal{F}_{\alpha}$ is the natural projection onto the first factor. Since $\mathcal{O}_{\alpha}^{w_{k}} \subset \operatorname{im}\left(\mathrm{q}_{k}\right)$, we have the surjective maps given below

$$
\mathrm{q}_{k}^{-1}\left(\mathcal{O}_{w^{\prime}, w^{\prime} w_{k}}^{w_{k}}\right) \stackrel{\mathrm{q}_{k}}{\longrightarrow} \mathcal{O}_{w^{\prime}, w^{\prime} w_{k}}^{w_{k}} \stackrel{\mathrm{pr}_{1}}{\longrightarrow} \mathcal{F}_{\nu_{w^{\prime}}} \quad \text { for } w^{\prime} \in \mathrm{W}_{\alpha} \backslash \mathrm{W} .
$$

By Lemma $2.1(\mathrm{vi})$, the map $\mathrm{q}_{k}^{-1}\left(\mathcal{O}_{w^{\prime}, w^{\prime} w_{k}}^{w_{k}}\right) \rightarrow \mathcal{F}_{\nu_{w^{\prime}}}$ is locally trivial with contractible fiber, and hence we have that

$$
\begin{aligned}
H_{*}^{\mathrm{G}_{\alpha}^{\Omega}}\left(\mathrm{q}_{k}^{-1}\left(\mathcal{O}_{w^{\prime}, w^{\prime} w_{k}}^{w_{k}}\right)\right) & \simeq H_{\mathrm{G}_{\alpha}^{\Omega}}^{*}\left(\mathcal{F}_{\nu_{w^{\prime}}}\right)\left\langle 2 \operatorname{dim}_{\mathbb{C}} \mathcal{Z}_{\nu_{w^{\prime}}, \nu_{w^{\prime} w_{k}}}^{w_{k}}\right\rangle \\
& \simeq \mathbf{H}\left[\chi_{1}\left(\nu_{w^{\prime}}\right), \ldots, \chi_{m}\left(\nu_{w^{\prime}}\right)\right]\left\langle 2 \operatorname{dim}_{\mathbb{C}} \mathcal{Z}_{\nu_{w^{\prime}}, \nu_{w^{\prime} w_{k}}}^{w_{k}}\right\rangle .
\end{aligned}
$$

Note that $\mathrm{q}_{k}^{-1}\left(\mathcal{O}_{w^{\prime}, w^{\prime} w_{k}}^{w_{k}}\right)$ is a dense open smooth subset of $\mathcal{Z}_{\nu_{w^{\prime}}, \nu_{w^{\prime} w_{k}}}^{w_{k}}$. Since $\mathcal{Z} \preceq k \backslash \mathcal{Z} \preceq k-1=$ $\mathrm{q}_{k}^{-1}\left(\mathcal{O}_{\alpha}^{w_{k}}\right)$, we get a long exact sequence $([5,(2.6 .10)])$

$$
\cdots \rightarrow H_{i}^{\mathrm{G}_{\alpha}^{\Omega}}(\mathcal{Z} \preceq k-1) \rightarrow H_{i}^{\mathrm{G}_{\alpha}^{\Omega}}(\mathcal{Z} \preceq k) \rightarrow H_{i}^{\mathrm{G}_{\alpha}^{\Omega}}\left(\mathrm{q}_{k}^{-1}\left(\mathcal{O}_{\alpha}^{w_{k}}\right)\right) \rightarrow H_{i+1}^{\mathrm{G}_{\alpha}^{\Omega}}(\mathcal{Z} \preceq k-1) \rightarrow \cdots
$$

for all $i \in \mathbb{Z}$. Since $H_{2 t+1}^{\mathrm{G}_{\alpha}^{\Omega}}\left(\mathcal{Z}^{\preceq k-1}\right)=H_{2 t+1}^{\mathrm{G}_{\alpha}^{\Omega}}\left(\mathrm{q}_{k}^{-1}\left(\mathcal{O}_{\alpha}^{w_{k}}\right)\right)=0$ for any $t \in \mathbb{Z}$ by $(2.2 .11)$ and (2.2.12), the following sequence is exact:

$$
0 \longrightarrow H_{*}^{\mathrm{G}_{\alpha}^{\Omega}}\left(\mathcal{Z}^{\preceq k-1}\right) \longrightarrow H_{*}^{\mathrm{G}_{\alpha}^{\Omega}}\left(\mathcal{Z}^{\preceq k}\right) \longrightarrow H_{*}^{\mathrm{G}_{\alpha}^{\Omega}}\left(\mathrm{q}_{k}^{-1}\left(\mathcal{O}_{\alpha}^{w_{k}}\right)\right) \longrightarrow 0 .
$$

By the isomorphisms (2.2.10) and (2.2.12), we conclude that $H_{*}^{\mathrm{G}_{\alpha}^{\Omega}}\left(\mathrm{q}_{k}^{-1}\left(\mathcal{O}_{\alpha}^{w_{k}}\right)\right)$ is a free $\mathfrak{R} \leq e_{-}$ module generated by the image of $\left[\mathcal{Z}_{\alpha}^{w_{k}}\right] \in H_{*}^{\mathrm{G}_{\alpha}^{\Omega}}\left(\mathcal{Z}^{\preceq k}\right)$. Hence the short exact sequence (2.2.13) splits and

$$
H_{*}^{\mathrm{G}_{\alpha}^{\Omega}}\left(\mathcal{Z}^{\prec k}\right)=H_{*}^{\mathrm{G}_{\alpha}^{\Omega}}\left(\mathcal{Z}^{\preceq k-1}\right) \oplus \mathfrak{R} \mathfrak{\alpha}^{\leq e} \star\left[\mathcal{Z}_{\alpha}^{w_{k}}\right], \quad H_{2 t+1}^{\mathrm{G}_{\alpha}^{\Omega}}\left(\mathcal{Z}^{\preceq k}\right)=0 .
$$

Hence the induction proceeds by (2.2.11) and we complete the proof of (ii).

We now proceed to prove the assertion (iii). For $k, k^{\prime}=1,2,3$, let $\mathbf{p}_{k, k^{\prime}}: \widetilde{\mathcal{F}}_{\alpha} \times \widetilde{\mathcal{F}}_{\alpha} \times$ $\widetilde{\mathcal{F}}_{\alpha} \rightarrow \widetilde{\mathcal{F}}_{\alpha} \times \widetilde{\mathcal{F}}_{\alpha}$ be the projection given by $\left(\left(x_{1}, F_{1}\right),\left(x_{2}, F_{2}\right),\left(x_{3}, F_{3}\right)\right) \mapsto\left(\left(x_{k}, F_{k}\right),\left(x_{k^{\prime}}, F_{k^{\prime}}\right)\right)$. Combining the assertion (ii) with the inclusion

$$
\mathbf{p}_{12}^{-1}\left(\mathcal{Z}_{\alpha}^{s_{j}}\right) \cap \mathbf{p}_{23}^{-1}\left(\mathcal{Z}_{\alpha}^{w}\right) \subset \mathbf{p}_{12}^{-1} \mathrm{q}_{\alpha}^{-1}\left(\overline{\mathcal{O}}_{\alpha}^{s_{j}}\right) \cap \mathbf{p}_{23}^{-1} \mathrm{q}_{\alpha}^{-1}\left(\overline{\mathcal{O}}_{\alpha}^{w}\right) \subset \mathbf{p}_{13}^{-1} \mathrm{q}_{\alpha}^{-1}\left(\overline{\mathcal{O}}_{\alpha}^{s_{j} w}\right) \subset \mathbf{p}_{13}^{-1}\left(\mathcal{Z}_{\alpha}^{\leq s_{j} w}\right),
$$


we have

$$
\left[\mathcal{Z}_{\alpha}^{s_{j}}\right] \star\left[\mathcal{Z}_{\alpha}^{w}\right]=c \star\left[\mathcal{Z}_{\alpha}^{s_{j} w}\right]+\sum_{w^{\prime}<s_{j} w} c_{w^{\prime}} \star\left[\mathcal{Z}_{\alpha}^{w^{\prime}}\right]
$$

for $c, c_{w^{\prime}} \in \mathfrak{R} \leq e$. To prove (3), it suffices to show $c=1$.

For $k, k^{\prime}=1,2,3$, let $\check{\mathbf{p}}_{k, k^{\prime}}: \mathcal{F}_{\alpha} \times \mathcal{F}_{\alpha} \times \mathcal{F}_{\alpha} \rightarrow \mathcal{F}_{\alpha} \times \mathcal{F}_{\alpha}$ be the natural projection given by $\left(F_{1}, F_{2}, F_{3}\right) \mapsto\left(F_{k}, F_{k^{\prime}}\right)$. Then it is known that $\ell\left(s_{j} w\right)=\ell(w)+1$ implies the following consequences:

$$
\left\{\begin{array}{c}
\left(\text { a) } \check{\mathbf{p}}_{12}^{-1}\left(\overline{\mathcal{O}}_{u, u s_{j}}^{s_{j}}\right) \cap \check{\mathbf{p}}_{23}^{-1}\left(\overline{\mathcal{O}}_{u s_{j}, u s_{j} w}^{w}\right) \cap \check{\mathbf{p}}_{13}^{-1}\left(\mathcal{O}_{u, u s_{j} w}^{s_{j} w}\right)=\check{\mathbf{p}}_{12}^{-1}\left(\mathcal{O}_{u, u s_{j}}^{s_{j}}\right) \cap \check{\mathbf{p}}_{23}^{-1}\left(\mathcal{O}_{u s_{j}, u s_{j} w}^{w}\right),\right. \\
\text { (b) } \check{\mathbf{p}}_{12}^{-1}\left(\mathcal{O}_{u, u s_{j}}^{s_{j}}\right) \text { and } \check{\mathbf{p}}_{23}^{-1}\left(\mathcal{O}_{u s_{j}, u s_{j} w}^{w}\right) \text { intersect transversally, } \\
\text { (c) The projection } \check{\mathbf{p}}_{13} \text { induces an isomorphism } \\
\check{\mathbf{p}}_{12}^{-1}\left(\mathcal{O}_{u, u s_{j}}^{s_{j}}\right) \cap \check{\mathbf{p}}_{23}^{-1}\left(\mathcal{O}_{u s_{j}, u s_{j} w}^{w}\right) \stackrel{\sim}{\longrightarrow} \mathcal{O}_{u, u s_{j} w}^{s_{j} w} .
\end{array}\right.
$$

Let us set

$$
\stackrel{\circ}{\mathcal{Z}}_{u^{\prime}, u^{\prime \prime}}^{u}=\mathrm{q}_{\alpha}^{-1}\left(\mathcal{O}_{u^{\prime}, u^{\prime \prime}}^{u}\right) \text { and } \stackrel{\circ}{\mathcal{Z}}_{\alpha}^{u}=\mathrm{q}_{\alpha}^{-1}\left(\mathcal{O}_{\alpha}^{u}\right) \quad \text { for } u, u^{\prime}, u^{\prime \prime} \in \mathrm{W} .
$$

Then, (2.2.15) (a) implies

$$
\mathbf{p}_{12}^{-1}\left(\mathcal{Z}_{\alpha}^{s_{j}}\right) \cap \mathbf{p}_{23}^{-1}\left(\mathcal{Z}_{\alpha}^{w}\right) \cap \mathbf{p}_{13}^{-1}\left(\stackrel{\circ}{\mathcal{Z}}_{\alpha}^{s_{j} w}\right)=\mathbf{p}_{12}^{-1}\left(\stackrel{\circ}{\mathcal{Z}}_{\alpha}^{s_{j}}\right) \cap \mathbf{p}_{23}^{-1}\left(\stackrel{\circ}{\mathcal{Z}}_{\alpha}^{w}\right) .
$$

Note that $\mathbf{p}_{12}^{-1}\left(\stackrel{\circ}{\mathcal{Z}}_{\alpha}^{s_{j}}\right)$ (resp. $\mathbf{p}_{23}^{-1}\left(\stackrel{\circ}{\mathcal{Z}}_{\alpha}^{w}\right)$ ) is a smooth dense open subset of $\mathbf{p}_{12}^{-1}\left(\mathcal{Z}_{\alpha}^{s_{j}}\right)$ (resp. $\left.\mathbf{p}_{23}^{-1}\left(\mathcal{Z}_{\alpha}^{w}\right)\right)$. Hence, by $(2.2 .13)$, (iii) is reduced to

$$
\left\{\begin{array}{l}
\text { (a) } \mathbf{p}_{13} \text { induces an isomorphism } \mathbf{p}_{12}^{-1}\left(\stackrel{\circ}{\mathcal{Z}}_{u, u s_{j}}^{s_{j}}\right) \cap \mathbf{p}_{23}^{-1}\left(\stackrel{\circ}{\mathcal{Z}}_{u s_{j}, u s_{j} w}^{w}\right) \stackrel{\circ}{\longrightarrow} \stackrel{\circ}{\mathcal{Z}}_{u, u s_{j} w}^{s_{j} w} . \\
\text { (b) } \mathbf{p}_{12}^{-1}\left(\stackrel{\circ}{\mathcal{Z}}_{u, u s_{j}}^{s_{j}}\right) \text { and } \mathbf{p}_{23}^{-1}\left(\stackrel{\circ}{\mathcal{Z}}_{u s_{j}, u s_{j} w}^{w}\right) \text { intersect transversally. }
\end{array}\right.
$$

Let us show first (2.2.17) (a). By using (2.2.15), it is enough to show that if $F_{1}$ and $F_{3}$ are strictly $x$-stable, then $F_{2}$ is strictly $x$ stable for $x \in \mathcal{E}_{\alpha}$ and $\left(F_{1}, F_{2}, F_{3}\right) \in \check{\mathbf{p}}_{12}^{-1}\left(\mathcal{O}_{u, u s_{j}}^{s_{j}}\right) \cap$ $\check{\mathbf{p}}_{23}^{-1}\left(\mathcal{O}_{u s_{j}, u s_{j} w}^{w}\right)$. Since $\check{\mathbf{p}}_{12}^{-1}\left(\mathcal{O}_{u, u s_{j}}^{s_{j}}\right) \cap \check{\mathbf{p}}_{23}^{-1}\left(\mathcal{O}_{u s_{j}, u s_{j} w}^{w}\right) \simeq \mathcal{O}_{u, u s_{j} w}^{s_{j} w}$ is a $G_{\alpha}$-orbit, we may assume that $\left(F_{1}, F_{2}, F_{3}\right)=\left(F_{u}, F_{u s_{j}}, F_{u s_{j} w}\right)$.

Since $x$ is nilpotent, it is enough to show that $x\left(F_{2}\right)_{k} \subset\left(F_{2}\right)_{k}$ for all $k$. We have $\left(F_{2}\right)_{k}=$ $\left(F_{1}\right)_{k}$ for $k \neq j, j+1$. It is therefore obvious that $x\left(F_{2}\right)_{k} \subset\left(F_{2}\right)_{k}$ for $k \neq j, j+1$. We have $\left(F_{2}\right)_{j}=\left(F_{2}\right)_{j-1}+\mathbb{F}_{u(j+1)}$, which implies that $x\left(F_{2}\right)_{j} \subset x\left(F_{1}\right)_{j-1}+x \mathbb{F}_{u(j+1)}$. Since $\mathbb{F}_{u(j+1)} \subset$ $\left(F_{1}\right)_{j+1} \cap\left(F_{u s_{j} w}\right)_{w^{-1}(j)}$, we have $x \mathbb{F}_{u(j+1)} \subset\left(F_{1}\right)_{j} \cap\left(F_{u s_{j} w}\right)_{w^{-1}(j)}$. On the other hand, we have $\left(F_{1}\right)_{j}=\left(F_{1}\right)_{j-1}+\mathbb{F}_{u j}$ and $\mathbb{F}_{u j} \cap\left(F_{u s_{j} w}\right)_{w^{-1}(j)}=\mathbb{F}_{u s_{j} w\left(w^{-1}(j+1)\right)} \cap\left(F_{u w s_{j}}\right)_{w^{-1}(j)}=0$ because $w^{-1}(j+1)>w^{-1}(j)$ by the assumption $s_{j} w>w$. It implies that $\left(F_{1}\right)_{j} \cap\left(F_{u s_{j} w}\right)_{w^{-1}(j)} \subset$ $\left(F_{1}\right)_{j-1}$. Hence we have $x\left(F_{2}\right)_{j} \subset\left(F_{2}\right)_{j-1}$. 
We have $\left(F_{2}\right)_{j+1}=\left(F_{2}\right)_{j}+\mathbb{F}_{u(j)} \subset\left(F_{2}\right)_{j}+\left(F_{1}\right)_{j}$. Hence $x\left(F_{2}\right)_{j+1} \subset x\left(F_{2}\right)_{j}+x\left(F_{1}\right)_{j} \subset$ $\left(F_{2}\right)_{j-1}$. Hence we complete the proof of (2.2.17) (a).

Let us show (2.2.17) (b). Since $\check{\mathbf{p}}_{12}^{-1}\left(\mathcal{O}_{u, u s_{j}}^{s_{j}}\right)$ and $\check{\mathbf{p}}_{23}^{-1}\left(\mathcal{O}_{u s_{j}, u s_{j} w}^{w}\right)$ intersect transversally, it is enough to show that the fiber of $\mathbf{p}_{1,2}^{-1}\left(\stackrel{\circ}{\mathcal{Z}}_{u, u s_{j}}^{s_{j}}\right) \rightarrow \check{\mathbf{p}}_{1,2}^{-1}\left(\mathcal{O}_{u, u s_{j}}^{s_{j}}\right)$ and the fiber of $\mathbf{p}_{2,3}^{-1}\left(\stackrel{\mathcal{Z}}{u s}_{u s_{j}, u s_{j} w}^{w}\right) \rightarrow$ $\check{\mathbf{p}}_{2,3}^{-1}\left(\mathcal{O}_{u s_{j}, u s_{j} w}^{w}\right)$ intersect transversally.

Let $\mathcal{T}^{1,2}$ (resp. $\left.\mathcal{T}^{2,3}\right)$ be the fiber of $\mathbf{p}_{1,2}^{-1}\left(\stackrel{\circ}{\mathcal{Z}}_{u, u s_{j}}^{s_{j}}\right) \rightarrow \check{\mathbf{p}}_{1,2}^{-1}\left(\mathcal{O}_{u, u s_{j}}^{s_{j}}\right)\left(\operatorname{resp} . \mathbf{p}_{2,3}^{-1}\left(\stackrel{\mathcal{Z}}{u s}_{u s_{j}, u s_{j} w}^{w}\right) \rightarrow\right.$ $\left.\check{\mathbf{p}}_{2,3}^{-1}\left(\mathcal{O}_{u s_{j}, u s_{j} w}^{w}\right)\right)$ at $\left(F_{u}, F_{u s_{j}}, F_{u s_{j} w}\right)$. We will show that $\mathcal{T}^{1,2}$ and $\mathcal{T}^{2,3}$ intersect transversally. Recall that $\mathfrak{e}_{v}$ is the fiber of $\widetilde{\mathcal{F}}_{\alpha} \rightarrow \mathcal{F}_{\alpha}$ at $F_{v}$ for $v \in \mathrm{W}$ (see (2.1.9)). Set $E_{1}=\mathfrak{e}_{u}, E_{2}=\mathfrak{e}_{u s_{j}}$ and $E_{3}=\mathfrak{e}_{u s_{j} w}$. Then we have

$$
\begin{aligned}
& \mathcal{T}^{1,2}=\left\{\left(v_{1}, v_{2}, v_{3}\right) \in E_{1} \oplus E_{2} \oplus E_{3} \mid v_{1}=v_{2}\right\}, \\
& \mathcal{T}^{2,3}=\left\{\left(v_{1}, v_{2}, v_{3}\right) \in E_{1} \oplus E_{2} \oplus E_{3} \mid v_{2}=v_{3}\right\} .
\end{aligned}
$$

In order to see that $\mathcal{T}^{1,2}$ and $\mathcal{T}^{2,3}$ intersect transversally in $E_{1} \oplus E_{2} \oplus E_{3}$, it is enough to show that

$$
E_{2}=\left(E_{1} \cap E_{2}\right)+\left(E_{2} \cap E_{3}\right) .
$$

Set $A(w)=\left\{\left(k, k^{\prime}\right) \mid 1 \leq k, k^{\prime} \leq m\right.$ and $\left.w^{-1}(k)>w^{-1}\left(k^{\prime}\right)\right\}$, and $A_{1}=A(u), A_{2}=A\left(u s_{j}\right)$

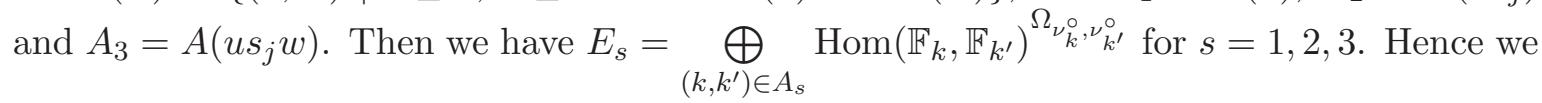
have reduced $(2.2 .18)$ to

$$
A_{2} \subset A_{1} \cup A_{3},
$$

which immediately follows from $A_{2} \backslash A_{1}=\{(u(j), u(j+1))\}$ and $(u(j), u(j+1)) \in A_{3}$. Here the last statement is a consequence of $w^{-1}(j+1)>w^{-1}(j)$. This completes the proof of $(2.2 .17)(\mathrm{b})$.

We now choose a set of generators of the convolution algebra $\mathfrak{R}(\alpha)$. By Lemma 2.3 and (2.2.10), we have an injective homomorphism

$$
\mathfrak{R} \leq e \simeq \bigoplus_{\nu \in I^{\alpha}} \mathbf{H}\left[\varkappa_{1}(\nu), \ldots, \varkappa_{m}(\nu)\right] \hookrightarrow \mathfrak{R}(\alpha) .
$$

For $\nu \in I^{\alpha}$, we define

$$
e(\nu)=\left[\mathcal{Z}_{\nu, \nu}^{e}\right]=\text { the image of } 1 \text { in } \mathbf{H}\left[\varkappa_{1}(\nu), \ldots, \varkappa_{m}(\nu)\right] \text { under (2.2.19), }
$$

For $\nu \in I^{\alpha}$ and $j=1, \ldots, m-1$, let $\tilde{\tau}_{j}=\left[\mathcal{Z}_{\alpha}^{s_{j}}\right] \in \mathfrak{R}(\alpha)$ and set

$$
\tau_{j}(\nu)=\left[\mathcal{Z}_{s_{j} \nu, \nu}^{s_{j}}\right]=e\left(s_{j} \nu\right) \star \tilde{\tau}_{j} \star e(\nu)=e\left(s_{j} \nu\right) \star \tilde{\tau}_{j}=\tilde{\tau}_{j} \star e(\nu) .
$$


Lemma 2.4. For $\nu=\left(\nu_{1}, \ldots, \nu_{m}\right) \in I^{\alpha}$, we have

$$
e(\nu) \in H_{\mathrm{d}_{\nu}}^{\mathrm{G}_{\alpha}^{\Omega}}\left(\mathcal{Z}_{\nu, \nu}\right), \quad \varkappa_{i}(\nu) \in H_{\mathrm{d}_{\nu}+2}^{\mathrm{G}_{\alpha}^{\Omega}}\left(\mathcal{Z}_{\nu, \nu}\right), \quad \tau_{j}(\nu) \in H_{\mathrm{d}_{s_{j}}+\mathrm{t}_{\nu, j}}^{\mathrm{G}^{\Omega}}\left(\mathcal{Z}_{s_{j} \nu, \nu}\right)
$$

where $\mathrm{d}_{\nu}=-2 \operatorname{dim}_{\mathbb{C}} \widetilde{\mathcal{F}}_{\nu}$ and $\mathrm{t}_{\nu, j}= \begin{cases}2\left(\ell_{\nu_{j}}-1\right) & \text { if } \nu_{j}=\nu_{j+1} \\ 2 h_{\nu_{j}, \nu_{j+1}} & \text { if } \nu_{j} \neq \nu_{j+1}\end{cases}$

Proof. It follows directly from (2.2.20) that $e(\nu) \in H_{\mathrm{d}_{\nu}}^{\mathrm{G}_{\alpha}^{\Omega}}\left(\mathcal{Z}_{\nu, \nu}\right)$ and $\varkappa_{i}(\nu) \in H_{\mathrm{d}_{\nu}+2}^{\mathrm{G}_{\alpha}^{\Omega}}\left(\mathcal{Z}_{\nu, \nu}\right)$. We focus on the assertion $\tau_{j}(\nu) \in H_{\mathrm{d}_{s_{j} \nu}+\mathrm{t}_{\nu, j}}^{\mathrm{G}^{\Omega}}\left(\mathcal{Z}_{s_{j} \nu, \nu}\right)$. Set

$$
\stackrel{\circ}{\mathcal{Z}}:=\mathrm{q}_{s_{j} \nu, \nu}^{-1}\left(\mathcal{O}_{s_{j} \nu, \nu}^{s_{j}}\right)
$$

Since $\stackrel{\circ}{\mathcal{Z}}$ is a dense open subset of $\mathcal{Z}_{s_{j} \nu, \nu}^{s_{j}}$, it suffices to show that

$$
\operatorname{dim}_{\mathbb{C}} \stackrel{\circ}{\mathcal{Z}}= \begin{cases}\operatorname{dim}_{\mathbb{C}} \widetilde{\mathcal{F}}_{\nu}-\ell_{\nu_{j}}+1 & \text { if } \nu_{j}=\nu_{j+1} \\ \operatorname{dim}_{\mathbb{C}} \widetilde{\mathcal{F}}_{s_{j} \nu}-h_{\nu_{j}, \nu_{j+1}} & \text { if } \nu_{j} \neq \nu_{j+1}\end{cases}
$$

By (2.1.10), we have

$$
\operatorname{dim}_{\mathbb{C}} \widetilde{\mathcal{F}}_{s_{j} \nu}=\operatorname{dim}_{\mathbb{C}} \mathcal{F}_{s_{j} \nu}+\sum_{k>k^{\prime}} \# \Omega_{\left(s_{j} \nu\right)_{k},\left(s_{j} \nu\right)_{k^{\prime}}}
$$

We now consider the following maps

$$
\stackrel{\circ}{\mathcal{Z}} \stackrel{\mathrm{q}_{s_{j} \nu, \nu}}{\longrightarrow} \mathcal{O}_{s_{j} \nu, \nu}^{s_{j}} \stackrel{\mathrm{pr}_{1}}{\longrightarrow} \mathcal{F}_{s_{j} \nu}
$$

where $\operatorname{pr}_{1}$ is the first projection. The fiber of the vector bundle $\stackrel{\circ}{\mathcal{Z}} \longrightarrow \mathcal{O}_{w s_{j}, w}^{s_{j}}$ is isomorphic to $\mathfrak{e}_{w s_{j}} \cap \mathfrak{e}_{w}$, and (2.1.10) implies that its dimension is equal to

$$
\sum_{k>k^{\prime},\left(k, k^{\prime}\right) \neq(j+1, j)} \# \Omega_{\left(s_{j} \nu\right)_{k},\left(s_{j} \nu\right)_{k^{\prime}}} .
$$

Hence we have

$$
\operatorname{dim}_{\mathbb{C}} \stackrel{\circ}{\mathcal{Z}}=\operatorname{dim}_{\mathbb{C}} \mathcal{O}_{s_{j} \nu, \nu}^{s_{j}}+\sum_{k>k^{\prime},\left(k, k^{\prime}\right) \neq(j+1, j)} \# \Omega_{\left(s_{j} \nu\right)_{k},\left(s_{j} \nu\right)_{k^{\prime}}}
$$

Together with (2.2.22) we obtain

$$
\operatorname{dim}_{\mathbb{C}} \stackrel{\circ}{\mathcal{Z}}-\operatorname{dim}_{\mathbb{C}} \tilde{\mathcal{F}}_{s_{j} \nu}=\operatorname{dim}_{\mathbb{C}} \mathcal{O}_{s_{j} \nu, \nu}^{s_{j}}-\operatorname{dim}_{\mathbb{C}} \mathcal{F}_{s_{j} \nu}-\# \Omega_{\left(s_{j} \nu\right)_{j+1},\left(s_{j} \nu\right)_{j}}
$$

Hence our claim follows from the fact

$$
\operatorname{dim}_{\mathbb{C}} \mathcal{O}_{s_{j} \nu, \nu}^{s_{j}}=\operatorname{dim}_{\mathbb{C}} \mathcal{F}_{s_{j} \nu}+\delta_{s_{j} \nu, \nu}
$$

in Lemma 2.1 (iii). 
2.3. Localization. Let us recall the localization theorem in equivariant cohomologies. We refer the reader to $[3,6]$.

Let $\mathbf{P}=H_{\mathrm{T}^{\Omega}}^{*}(\mathrm{pt})$ and recall the ring $\mathbf{S}=H_{\mathrm{G}_{\alpha}^{\Omega}}^{*}(\mathrm{pt})$ given in (2.2.1). We have

$$
\mathbf{P} \simeq \mathbf{H} \otimes H_{\mathrm{T}}^{*}(\mathrm{pt}) \simeq \mathbf{H}\left[\chi_{1}, \ldots, \chi_{m}\right]
$$

with $\operatorname{deg} \chi_{i}=2$ for $i=1, \ldots, m$. The action of $\mathrm{W}$ on $\mathrm{T}$ induces the action of $\mathrm{W}$ on $\mathbf{P}$; i.e.,

$$
w\left(f\left(\chi_{1}, \ldots, \chi_{m}\right)\right)=f\left(\chi_{w(1)}, \ldots, \chi_{w(m)}\right)
$$

for $w \in \mathrm{W}, f\left(\chi_{1}, \ldots, \chi_{m}\right) \in \mathbf{H}\left[\chi_{1}, \ldots, \chi_{m}\right]$. Then the group morphism $\mathrm{T} \rightarrow \mathrm{G}_{\alpha}$ induces the homomorphism $\mathbf{S}=H_{\mathrm{G}_{\alpha}^{\Omega}}^{*}(\mathrm{pt}) \rightarrow H_{\mathrm{T}^{\Omega}}^{*}(\mathrm{pt})$ and $\mathbf{S}$ can be identified with

$$
\mathbf{S} \simeq \mathbf{H}\left[\chi_{1}, \ldots, \chi_{m}\right]^{\mathrm{W}_{\alpha}} \hookrightarrow \mathbf{P}
$$

where $\mathbf{H}\left[\chi_{1}, \ldots, \chi_{m}\right]^{\mathrm{W}_{\alpha}}$ is the set of $\mathrm{W}_{\alpha}$-invariant polynomials in $\mathbf{H}\left[\chi_{1}, \ldots, \chi_{m}\right]$. Let $\mathbf{K}$ be the fraction field of $\mathbf{P}$ :

$$
\mathbf{K}=\mathbb{C}\left(\chi_{1}, \ldots, \chi_{m}, \hbar_{a}(a \in \Omega)\right)
$$

and consider $\mathbf{P}$ as a subring of $\mathbf{K}$.

Let $X$ be a quasi-projective $\mathrm{T}^{\Omega}$-variety. Then the inclusion $\iota: X^{\mathrm{T}^{\Omega}} \hookrightarrow X$ induces isomorphisms (localization theorem):

$$
\mathbf{K} \otimes_{\mathbf{P}} H_{*}^{\mathrm{T}^{\Omega}}\left(X^{\mathrm{T}^{\Omega}}\right) \underset{\iota_{*}}{\sim} \mathbf{K} \otimes_{\mathbf{P}} H_{*}^{\mathrm{T}^{\Omega}}(X)
$$

and

$$
\mathbf{K} \otimes \mathbf{P} H_{\mathrm{T}^{\Omega}}^{*}(X) \underset{\iota^{*}}{\sim} \mathbf{K} \otimes \mathbf{P} H_{\mathrm{T}^{\Omega}}^{*}\left(X^{\mathrm{T}^{\Omega}}\right) .
$$

Let $\mathfrak{t}^{\Omega}:=\mathfrak{t} \oplus \mathfrak{h}^{\Omega}$ be the Lie algebra of the group $\mathrm{T}^{\Omega}=\mathrm{T} \times \mathrm{H}^{\Omega}$. For a finite-dimensional weight module $M$ over $\mathfrak{t}^{\Omega}$, set

$$
\mathfrak{d}(M)=\prod_{\mu} \mu^{\operatorname{dim}_{\mathbb{C}} M_{\mu}} \in \mathbf{P},
$$

where $M=\bigoplus_{\mu} M_{\mu}$ is the weight space decomposition of $M$.

Assume that

(2.3.3) the fixed point set $X^{\mathrm{T}^{\Omega}}$ is finite and consists of smooth points of $X$.

For a smooth $\mathrm{T}^{\Omega}$-fixed point $p$, the equivariant Euler class $\mathrm{eu}(X, p)$ is by definition the image of $1 \in \mathbf{P}$ by the composition $\mathbf{P} \simeq H_{\mathrm{T}^{\Omega}}^{*}(p) \longrightarrow H_{\mathrm{T}^{\Omega}}^{*}(X)\left\langle 2 \operatorname{dim}_{\mathbb{C}} X\right\rangle \longrightarrow H_{\mathrm{T}^{\Omega}}^{*}(p)\left\langle 2 \operatorname{dim}_{\mathbb{C}} X\right\rangle \simeq$ $\mathbf{P}\left\langle 2 \operatorname{dim}_{\mathbb{C}} X\right\rangle$ where the first arrow is the Gysin map. Then we have (see e.g. [3])

$$
\mathrm{eu}(X, p)=\mathfrak{d}\left(T_{p} X\right)
$$


The localization theorem (see e.g. [3]) says

$$
[X]=\sum_{p \in X^{\mathrm{T}^{\Omega}}} \operatorname{eu}(X, p)^{-1}[p] \quad \text { in } \mathbf{K} \otimes_{\mathbf{P}} H_{*}^{\mathrm{T}^{\Omega}}(X) .
$$

Note that $\mathrm{eu}(X, p)$ never vanishes. Note also that, under the condition (2.3.3), the Gysin morphism induces an isomorphism:

$$
\mathbf{K} \otimes_{\mathbf{P}} H_{\mathrm{T}^{\Omega}}^{*}\left(X^{\mathrm{T}^{\Omega}}\right) \stackrel{\sim}{\sim} \mathbf{K} \otimes_{\mathbf{P}} H_{\mathrm{T}^{\Omega}}^{*}(X)\left\langle 2 \operatorname{dim}_{\mathbb{C}} X\right\rangle .
$$

We now apply the localization theorem to $\mathrm{T}^{\Omega}$-varieties $\widetilde{\mathcal{F}}_{\alpha}$ and $\mathcal{Z}_{\alpha}$. By $[4$, Proposition 1.2.1] and Lemma $2.1(1)$, the sets $\widetilde{\mathcal{F}}_{\alpha}^{\mathrm{T}^{\Omega}}$ and $\mathcal{Z}_{\alpha}^{\mathrm{T}^{\Omega}}$ of $\mathrm{T}^{\Omega}$-fixed points are given by

$$
\begin{aligned}
& \widetilde{\mathcal{F}}_{\alpha}^{\mathrm{T}^{\Omega}}=\left\{\left(0, F_{w}\right) \mid w \in \mathrm{W}\right\} \subset \widetilde{\mathcal{F}}_{\alpha}, \quad \widetilde{\mathcal{F}}_{\nu}^{\mathrm{T}^{\Omega}}=\widetilde{\mathcal{F}}_{\alpha}^{\mathrm{T}^{\Omega}} \cap \widetilde{\mathcal{F}}_{\nu}, \\
& \mathcal{Z}_{\alpha}^{\mathrm{T}^{\Omega}}=\left\{\left(0, F_{w, w^{\prime}}\right) \mid w, w^{\prime} \in \mathrm{W}\right\} \subset \mathcal{Z}_{\alpha}, \quad \mathcal{Z}_{\nu, \nu^{\prime}}^{\mathrm{T}^{\Omega}}=\mathcal{Z}_{\alpha}^{\mathrm{T}^{\Omega}} \cap \mathcal{Z}_{\nu, \nu^{\prime}}
\end{aligned}
$$

for $\nu, \nu^{\prime} \in I^{\alpha}$. Then we have $\mathbf{K}$-module isomorphisms by (2.3.1) and (2.3.6)

$$
\begin{aligned}
& \mathbf{K} \otimes_{\mathbf{P}} H_{\mathrm{T}^{\Omega}}^{*}\left(\widetilde{\mathcal{F}}_{\nu}^{\mathrm{T}^{\Omega}}\right) \stackrel{\sim}{\sim} \mathbf{K} \otimes_{\mathbf{P}} H_{\mathrm{T}^{\Omega}}^{*}\left(\widetilde{\mathcal{F}}_{\nu}\right)\left\langle 2 \operatorname{dim}_{\mathbb{C}} \widetilde{\mathcal{F}}_{\nu}\right\rangle, \\
& \mathbf{K} \otimes_{\mathbf{P}} H_{*}^{\mathrm{T}^{\Omega}}\left(\mathcal{Z}_{\nu, \nu^{\prime}}^{\mathrm{T}^{\Omega}}\right) \stackrel{\sim}{\longrightarrow} \otimes_{\mathbf{P}} H_{*}^{\mathrm{T}^{\Omega}}\left(\mathcal{Z}_{\nu, \nu^{\prime}}\right),
\end{aligned}
$$

which yields

$$
\mathbf{K} \otimes_{\mathbf{P}} H_{\mathrm{T}^{\Omega}}^{*}\left(\widetilde{\mathcal{F}}_{\nu}\right) \simeq \bigoplus_{w \in \mathrm{W}} \mathbf{K} \zeta_{w}, \quad \mathbf{K} \otimes_{\mathbf{P}} H_{*}^{\mathrm{T}^{\Omega}}\left(\mathcal{Z}_{\nu, \nu^{\prime}}\right) \simeq \bigoplus_{w, w^{\prime} \in \mathrm{W}} \mathbf{K} \zeta_{w, w^{\prime}}
$$

Here, $\zeta_{w}\left(\operatorname{resp} . \zeta_{w, w^{\prime}}\right)$ is the element in $H_{\mathrm{T}^{\Omega}}^{*}\left(\widetilde{\mathcal{F}}_{\nu}\right)\left(\right.$ resp. $\left.H_{*}^{\mathrm{T}^{\Omega}}\left(\mathcal{Z}_{\nu, \nu^{\prime}}\right)\right)$ which is the image of $\left(0, F_{w}\right) \in H_{\mathrm{T}^{\Omega}}^{*}\left(\widetilde{\mathcal{F}}_{\nu}^{\mathrm{T}^{\Omega}}\right)\left(\operatorname{resp} .\left(0, F_{w, w^{\prime}}\right) \in H_{*}^{\mathrm{T}^{\Omega}}\left(\mathcal{Z}_{\nu, \nu^{\prime}}^{\mathrm{T}^{\Omega}}\right)\right)$ under the isomorphisms $(2.3 .7)$. We have the following injective $\mathbf{P}$-module homomorphisms [20, (2.3)]

$$
\begin{gathered}
\Psi_{\nu}: H_{\mathrm{G}_{\alpha}^{\Omega}}^{*}\left(\widetilde{\mathcal{F}}_{\nu}\right) \hookrightarrow \mathbf{K} \otimes \mathbf{P} H_{\mathrm{T}^{\Omega}}^{*}\left(\widetilde{\mathcal{F}}_{\nu}\right), \\
\Phi_{\nu, \nu^{\prime}}: H_{*}^{\mathrm{G}_{\alpha}^{\Omega}}\left(\mathcal{Z}_{\nu, \nu^{\prime}}\right) \hookrightarrow \mathbf{K} \otimes_{\mathbf{P}} H_{*}^{\mathrm{T}^{\Omega}}\left(\mathcal{Z}_{\nu, \nu^{\prime}}\right)
\end{gathered}
$$

for $\nu, \nu^{\prime} \in I^{\alpha}$. Let $\Psi_{\alpha}=\sum_{\nu \in I^{\alpha}} \Psi_{\nu}$ and $\Phi_{\alpha}=\sum_{\nu, \nu^{\prime} \in I^{\alpha}} \Phi_{\nu, \nu^{\prime}}$. Then we obtain the following commutative diagram:

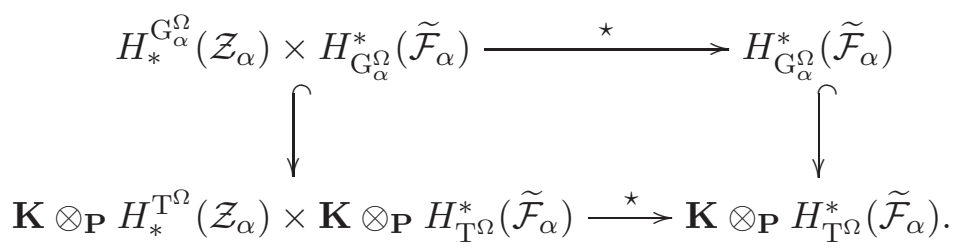

Here, $\star$ is the convolution product. 
Set

$$
\begin{aligned}
\Lambda_{w}=\operatorname{eu}\left(\widetilde{\mathcal{F}}_{\alpha},\left(0, F_{w}\right)\right) & \text { for } w \in \mathrm{W} \text { and, } \\
\Lambda_{w, w^{\prime}}^{s_{j}}=\operatorname{eu}\left(\mathcal{Z}_{w, w^{\prime}}^{s_{j}},\left(0, F_{w, w^{\prime}}\right)\right) & \text { for } j=1, \ldots, m-1 \text { and } \\
& w, w^{\prime} \in \mathrm{W} \text { such that } w^{\prime}=w, w s_{j} \text { and } \nu_{w}=\nu_{w^{\prime} s_{j}} .
\end{aligned}
$$

Then $\Lambda_{w}$ and $\Lambda_{w, w^{\prime}}^{s_{j}}$ are elements of $\mathbf{P}$ of degree $2 \operatorname{dim}_{\mathbb{C}} \widetilde{\mathcal{F}}_{\nu_{w}}$ and $2 \operatorname{dim} \mathcal{Z}_{\nu_{w}, \nu_{w^{\prime}}}^{s_{j}}$, respectively. Note that $\widetilde{\mathcal{F}}_{\alpha}$ and $\mathcal{Z}_{w, w^{\prime}}^{s_{j}}$ are smooth varieties with finitely many $\mathrm{T}^{\Omega}$-fixed points (see Lemma 2.2).

By Lemma 2.1 and (2.3.5), we obtain the following lemma (see also [20, Lemma 2.17, Lemma 2.19]).

\section{Lemma 2.5.}

(i) For $\nu \in I^{\alpha}$ and $f \in \mathbf{H}\left[x_{1}, \ldots, x_{m}\right]$, we have

$$
\Psi_{\nu}\left(f\left(\chi_{1}(\nu), \ldots, \chi_{m}(\nu)\right)\right)=\sum_{w \in \mathrm{W}(\nu)} f\left(\chi_{w(1)}, \ldots, \chi_{w(m)}\right) \Lambda_{w}^{-1} \zeta_{w},
$$

where $\mathrm{W}(\nu):=\left\{w \in \mathrm{W} \mid F_{w} \in \mathcal{F}_{\nu}\right\}=\left\{w \in \mathrm{W} \mid \nu_{w}=\nu\right\}$.

(ii) For $\nu \in I^{\alpha}$ and $f \in \mathbf{H}\left[x_{1}, \ldots, x_{m}\right]$,

$$
\Phi_{\nu, \nu}\left(f\left(\varkappa_{1}(\nu), \ldots, \varkappa_{m}(\nu)\right)\right)=\sum_{w \in \mathrm{W}(\nu)} f\left(\chi_{w(1)}, \ldots, \chi_{w(m)}\right) \Lambda_{w}^{-1} \zeta_{w, w},
$$

(iii) For $j=1, \ldots, m-1$ and $\nu \in I^{\alpha}$,

$$
\Phi_{\alpha}\left(\tau_{j}(\nu)\right)= \begin{cases}\sum_{w \in \mathrm{W}(\nu)} \Lambda_{w s_{j}, w}^{s_{j}}{ }^{-1} \zeta_{w s_{j}, w} & \text { if } s_{j} \nu \neq \nu, \\ \sum_{w \in \mathrm{W}(\nu)}\left(\Lambda_{w, w}^{s_{j}}{ }^{-1} \zeta_{w, w}+\Lambda_{w s_{j}, w}^{s_{j}}{ }^{-1} \zeta_{w s_{j}, w}\right) & \text { if } s_{j} \nu=\nu .\end{cases}
$$

(iv) For $w, w^{\prime}, w^{\prime \prime} \in \mathrm{W}$, we have

$$
\zeta_{w, w^{\prime}} \star \zeta_{w^{\prime \prime}}=\delta_{w^{\prime}, w^{\prime \prime}} \Lambda_{w^{\prime \prime}} \zeta_{w} .
$$

Proof. (i) For any $w \in W(\nu)$, let $R_{w}$ be the map $\mathbf{K} \otimes_{\mathbf{P}} H_{\mathrm{T}^{\Omega}}^{*}\left(\widetilde{\mathcal{F}}_{\nu}\right) \rightarrow \mathbf{K} \otimes_{\mathbf{P}} H_{\mathrm{T}^{\Omega}}^{*}\left(\left(0, F_{w}\right)\right) \simeq \mathbf{K}$ induced by the inclusion $\left(0, F_{w}\right) \hookrightarrow \widetilde{\mathcal{F}}_{w}$. Then the map

$$
\mathbf{K} \otimes \mathbf{P} H_{\mathrm{T}^{\Omega}}^{*}\left(\widetilde{\mathcal{F}}_{\nu}\right) \stackrel{\oplus R_{w}}{\longrightarrow} \underset{w \in W(\nu)}{\bigoplus} \mathbf{K} \otimes_{\mathbf{P}} H_{\mathrm{T}^{\Omega}}^{*}\left(\left(0, F_{w}\right)\right) \simeq H_{\mathrm{T}^{\Omega}}^{*}\left(\widetilde{\mathcal{F}}_{\nu}^{\mathrm{T}^{\Omega}}\right)
$$

is an isomorphism (see (2.3.2)). Then (i) follows from

$$
R_{w}\left(\Psi_{\nu}\left(f\left(\chi_{1}(\nu), \ldots, \chi_{m}(\nu)\right)\right)\right)=f\left(\chi_{w(1)}, \ldots, \chi_{w(m)}\right)
$$

(see (2.2.4)) and $R_{w}\left(\zeta_{w^{\prime}}\right)=\delta_{w, w^{\prime}} \Lambda_{w}$.

(ii) is similarly proved. 
(iii) immediately follows from (2.3.5) with $X=\mathcal{Z}_{s_{j} \nu, \nu}^{s_{j}}$.

(iv) It is obvious that $\zeta_{w, w^{\prime}} \star \zeta_{w^{\prime \prime}}=0$ as soon as $w^{\prime} \neq w^{\prime \prime}$. Hence we have

$$
\zeta_{w}=\zeta_{w, w^{\prime}} \star\left[\widetilde{\mathcal{F}}_{\alpha}\right]=\zeta_{w, w^{\prime}} \star\left(\sum_{w^{\prime \prime} \in W} \Lambda_{w^{\prime \prime}}^{-1} \zeta_{w^{\prime \prime}}\right)=\zeta_{w, w^{\prime}} \star\left(\Lambda_{w^{\prime}}^{-1} \zeta_{w^{\prime}}\right) .
$$

Here the second equality follows from (2.3.5).

Recall that $\mathfrak{e}_{w}$ is the fiber of the vector bundle $\widetilde{\mathcal{F}}_{\alpha} \rightarrow \mathcal{F}_{\alpha}$ at $F_{w}$ (see (2.1.9)). Hence we have

$$
\Lambda_{w}=\operatorname{eu}\left(\widetilde{\mathcal{F}}_{\nu_{w}},\left(0, F_{w}\right)\right)=\operatorname{eu}\left(\mathcal{F}_{\nu_{w}}, F_{w}\right) \mathfrak{d}\left(\mathfrak{e}_{w}\right) .
$$

Let $w, w^{\prime} \in \mathrm{W}$ such that $F_{w^{\prime}, w} \in \overline{\mathcal{O}}_{\alpha}^{s_{j}}$, 1.e., $w^{\prime}=w, w s_{j}$ and $\nu_{w}=\nu_{w^{\prime} s_{j}}$ (see Lemma 2.1). Then $\mathcal{Z}_{\alpha}^{s_{j}} \rightarrow \overline{\mathcal{O}}_{\alpha}^{s_{j}}$ is a vector bundle and its fiber at $F_{w, w^{\prime}}$ is $\mathfrak{e}_{w} \cap \mathfrak{e}_{w s_{j}}$ (see Lemma 2.2). Hence we have

$$
\begin{aligned}
\Lambda_{w, w^{\prime}}^{s_{j}} & =\operatorname{eu}\left(\mathcal{Z}_{\alpha}^{s_{j}},\left(0, F_{w, w^{\prime}}\right)\right) \\
& =\operatorname{eu}\left(\overline{\mathcal{O}}_{\alpha}^{s_{j}}, F_{w, w^{\prime}}\right) \mathfrak{d}\left(\mathfrak{e}_{w} \cap \mathfrak{e}_{w s_{j}}\right) .
\end{aligned}
$$

In the following lemma, we compute the quotients $\Lambda_{w, w}^{s_{j}{ }^{-1}} \Lambda_{w}$ and $\Lambda_{w, w s_{j}}^{s_{j}} \Lambda_{w}$ of the equivariant Euler classes in order to describe explicitly the actions of $e(\nu), \varkappa_{k}(\nu)$ and $\tau_{t}(\nu)$ on $\mathfrak{P o l}(\alpha)$. The polynomials $\mathcal{P}_{i}$ and $\mathcal{Q}_{i, j}$ given in (1.2.6) arise naturally in the course of computation.

Lemma 2.6. Let $w \in \mathrm{W}$ and $j=1, \ldots, m-1$. Set $\nu=\nu_{w}$ and write $\nu=\left(\nu_{1}, \nu_{2}, \ldots, \nu_{m}\right)$.

(i) If $\nu \neq s_{j} \nu$, then

$$
\Lambda_{w, w s_{j}}^{s_{j}}{ }^{-1} \Lambda_{w}=\prod_{a \in \Omega_{\nu_{j+1}, \nu_{j}}}\left(\chi_{w(j)}-\chi_{w(j+1)}+\hbar_{a}\right),
$$

(ii) If $\nu=s_{j} \nu$, then

$$
\Lambda_{w, w^{\prime}}^{s_{j}}{ }^{-1} \Lambda_{w}=(-1)^{\delta_{w, w^{\prime}}} \frac{\prod_{a \in \Omega_{\nu_{j}, \nu_{j}}}\left(\chi_{w(j)}-\chi_{w(j+1)}+\hbar_{a}\right)}{\chi_{w(j)}-\chi_{w(j+1)}},
$$

where $w^{\prime}=w$ or $w^{\prime}=w s_{j}$.

Proof. By (2.1.10), we have

$$
\frac{\mathfrak{e}_{w}}{\mathfrak{e}_{w} \cap \mathfrak{e}_{w s_{j}}} \simeq \bigoplus_{a \in \Omega_{\nu_{j+1}, \nu}} \operatorname{Hom}\left(\mathbb{F}_{w(j+1)}, \mathbb{F}_{w(j)}\right)
$$

as a $\mathfrak{t}^{\Omega}$-module. Hence we have

$$
\mathfrak{d}\left(\frac{\mathfrak{e}_{w}}{\mathfrak{e}_{w} \cap \mathfrak{e}_{w s_{j}}}\right)=\prod_{a \in \Omega_{\nu_{j+1}, \nu_{j}}}\left(\chi_{w(j)}-\chi_{w(j+1)}+\hbar_{a}\right) .
$$


By (2.3.11) and (2.3.12), we have

$$
\begin{aligned}
\Lambda_{w, w^{\prime}}^{s_{j}}{ }^{-1} \Lambda_{w} & =\frac{\mathrm{eu}\left(\mathcal{F}_{w}, F_{w}\right)}{\operatorname{eu}\left(\overline{\mathcal{O}}_{w, w^{\prime}}^{s_{j}}, F_{w, w^{\prime}}\right)} \frac{\mathfrak{d}\left(\mathfrak{e}_{w}\right)}{\mathfrak{d}\left(\mathfrak{e}_{w} \cap \mathfrak{e}_{w s_{j}}\right)} \\
& =\frac{\mathrm{eu}\left(\mathcal{F}_{w}, F_{w}\right)}{\operatorname{eu}\left(\overline{\mathcal{O}}_{w, w^{\prime}}^{s_{j}}, F_{w, w^{\prime}}\right)} \prod_{a \in \Omega_{\nu_{j+1}, \nu_{j}}}\left(\chi_{w(j)}-\chi_{w(j+1)}+\hbar_{a}\right) .
\end{aligned}
$$

(i) Assume that $\nu \neq s_{j} \nu$. Then $w^{\prime}=w s_{j}$ and $\overline{\mathcal{O}}_{w, w s_{j}}^{s_{j}} \stackrel{\sim}{\longrightarrow} \mathcal{F}_{\nu_{w}}$ by Lemma $2.1(\mathrm{v})$. Hence we obtain (i).

(ii) Assume that $\nu_{w}=s_{j} \nu_{w}$, and $w^{\prime}=w, w s_{j}$. Recall that $B_{\alpha, w}=\left\{g \in G_{\alpha} \mid g F_{w}=F_{w}\right\}$ and $\mathfrak{b}_{\alpha, w}$ is its Lie algebra. The morphism $\overline{\mathcal{O}}_{w, w^{\prime}}^{s_{j}} \rightarrow \mathcal{F}_{w}$ is a $\mathbb{P}^{1}$-bundle and the tangent space of the fiber at $F_{w, w^{\prime}}$ is isomorphic to

$$
\frac{\mathfrak{b}_{\alpha, w}+\mathfrak{b}_{\alpha, w s_{j}}}{\mathfrak{b}_{\alpha, w^{\prime}}} \simeq \begin{cases}\operatorname{Hom}\left(\mathbb{F}_{w(j)}, \mathbb{F}_{w(j+1)}\right) & \text { if } w^{\prime}=w \\ \operatorname{Hom}\left(\mathbb{F}_{w(j+1)}, \mathbb{F}_{w(j)}\right) & \text { if } w^{\prime}=w s_{j}\end{cases}
$$

by (2.1.11). Hence we obtain

$$
\frac{\mathrm{eu}\left(\overline{\mathcal{O}}_{w, w^{\prime}}^{s_{j}}, F_{w, w^{\prime}}\right)}{\mathrm{eu}\left(\mathcal{F}_{w}, F_{w}\right)}= \begin{cases}\chi_{w(j+1)}-\chi_{w(j)} & \text { if } w^{\prime}=w \\ \chi_{w(j)}-\chi_{w(j+1)} & \text { if } w^{\prime}=w s_{j}\end{cases}
$$

which implies (ii).

We now describe explicitly the $\mathfrak{R}(\alpha)$-module structure of $\mathfrak{P o r}(\alpha)$. Recall that for $f \in$ $\mathbf{H}\left[x_{1}, \ldots, x_{m}\right]$ and $\nu \in I^{\alpha}$, we denote by $f(\nu)$ the element $f\left(\chi_{1}(\nu), \ldots, \chi_{m}(\nu)\right) \in \mathfrak{P o r}(\alpha)$. Recall also that $(w f)\left(x_{1}, \ldots, x_{m}\right)=f\left(x_{w(1)}, \ldots, x_{w(m)}\right)$ for $w \in \mathrm{W}$. The actions of $e(\nu)$, $\varkappa_{k}(\nu), \tau_{t}(\nu) \in \mathfrak{R}(\alpha)\left(k=1, \ldots, m, t=1, \ldots, m-1, \nu \in I^{\alpha}\right)$ on $\mathfrak{P o r}(\alpha)$ are given explicitly in the following proposition.

\section{Proposition 2.7.}

(i) $\mathfrak{P o l}(\alpha)$ is a faithful $\mathfrak{R}(\alpha)$-module.

(ii) Let $f \in \mathbf{H}\left[\chi_{1}, \ldots, \chi_{m}\right]$, and $\nu, \nu^{\prime} \in I^{\alpha}$.

(a) For $k=1, \ldots, m$, we have

$$
e(\nu) \star f\left(\nu^{\prime}\right)=\left\{\begin{array}{ll}
f(\nu) & \text { if } \nu=\nu^{\prime}, \\
0 & \text { if } \nu \neq \nu^{\prime},
\end{array} \quad \varkappa_{k}(\nu) \star f\left(\nu^{\prime}\right)= \begin{cases}\chi_{k}(\nu) f(\nu) & \text { if } \nu=\nu^{\prime}, \\
0 & \text { if } \nu \neq \nu^{\prime} .\end{cases}\right.
$$

(b) For $j=1, \ldots, m-1$ and $\nu=\left(\nu_{1}, \ldots, \nu_{m}\right)$, we have 


$$
\tilde{\tau}_{j} \star f(\nu)= \begin{cases}\left(\prod_{a \in \Omega_{\nu_{j}, \nu_{j+1}}}\left(\chi_{j}\left(s_{j} \nu\right)-\chi_{j+1}\left(s_{j} \nu\right)+\hbar_{a}\right)\right)\left(s_{j} f\right)\left(s_{j} \nu\right) & \text { if } s_{j} \nu \neq \nu \\ \left(\prod_{a \in \Omega_{\nu_{j}, \nu_{j}}}\left(\chi_{j}(\nu)-\chi_{j+1}(\nu)+\hbar_{a}\right)\right)\left(\partial_{j} f\right)(\nu) & \text { if } s_{j} \nu=\nu .\end{cases}
$$

Proof. (i) Our assertion follows from Lemma 2.5 (iv).

(ii) Since the assertion (a) is straightforward, we shall prove the assertion (b). Since the diagram (2.3.10) is commutative, it suffices to show that

$$
\Phi_{\alpha}\left(\tilde{\tau}_{j}\right) \star \Psi_{\alpha}(f(\nu))=\Psi_{\alpha}(g)
$$

where $g= \begin{cases}\left(\prod_{a \in \Omega_{\nu_{j}, \nu_{j}+1}}\left(\chi_{j}\left(s_{j} \nu\right)-\chi_{j+1}\left(s_{j} \nu\right)+\hbar_{a}\right)\right)\left(s_{j} f\right)\left(s_{j} \nu\right) & \text { if } s_{j} \nu \neq \nu \\ \left.\prod_{a \in \Omega_{\nu_{j}, \nu_{j}}}\left(\chi_{j}(\nu)-\chi_{j+1}(\nu)+\hbar_{a}\right)\right)\left(\partial_{j} f\right)(\nu) & \text { if } s_{j} \nu=\nu\end{cases}$

By Lemma 2.5, we have

$$
\begin{aligned}
\Phi_{\alpha}\left(\tilde{\tau}_{j}\right) \star \Psi_{\alpha}(f(\nu)) & =\sum_{w \in \mathrm{W}}\left(\delta_{\nu_{w}, \nu_{w s_{j}}} \Lambda_{w, w}^{s_{j}}{ }^{-1} \zeta_{w, w}+\Lambda_{w s_{j}, w}^{s_{j}}{ }^{-1} \zeta_{w s_{j}, w}\right) \star \sum_{w \in \mathrm{W}(\nu)}(w f) \Lambda_{w}^{-1} \zeta_{w} \\
& =\sum_{w \in \mathrm{W}(\nu)}\left(\delta_{\nu_{w}, \nu_{w s_{j}}} \Lambda_{w, w}^{s_{j}}{ }^{-1}(w f) \zeta_{w}+\Lambda_{w s_{j}, w}^{s_{j}}{ }^{-1}(w f) \zeta_{w s_{j}}\right) .
\end{aligned}
$$

Suppose that $\nu \neq s_{j} \nu$. Then, by Lemma 2.6 and (2.3.14), we have

$$
\begin{aligned}
\Phi_{\alpha}\left(\tilde{\tau}_{j}\right) \star \Psi_{\alpha}(f(\nu)) & =\sum_{w \in \mathrm{W}(\nu)} \Lambda_{w s_{j}, w}^{s_{j}}(w f) \zeta_{w s_{j}} \\
& =\sum_{w \in \mathrm{W}\left(s_{j} \nu\right)} \Lambda_{w, w s_{j}}^{s_{j}}{ }^{-1} \Lambda_{w}\left(w s_{j} f\right)\left(\Lambda_{w}^{-1} \zeta_{w}\right) \\
& =\sum_{w \in \mathrm{W}\left(s_{j} \nu\right)}\left(\prod_{a \in \Omega_{\nu_{j}, \nu_{j+1}}}\left(\chi_{w(j)}-\chi_{w(j+1)}+\hbar_{a}\right)\right)\left(w s_{j} f\right) \Lambda_{w}^{-1} \zeta_{w} \\
& \left.=\Psi_{\alpha}\left(\prod_{a \in \Omega_{\nu_{j}, \nu_{j+1}}}\left(\chi_{j}\left(s_{j} \nu\right)-\chi_{j+1}\left(s_{j} \nu\right)+\hbar_{a}\right)\right)\left(s_{j} f\right)\left(s_{j} \nu\right)\right) .
\end{aligned}
$$

Here the last equality follows from Lemma 2.5 (i).

We now assume that $\nu=s_{j} \nu$. By Lemma 2.6 and (2.3.14), we obtain 


$$
\begin{aligned}
\Phi_{\alpha}\left(\tilde{\tau}_{j}\right) \star \Psi_{\alpha}(f(\nu)) & =\sum_{w \in \mathrm{W}(\nu)}\left(\Lambda_{w, w}^{s_{j}}{ }^{-1}(w f) \zeta_{w}+\Lambda_{w s_{j}, w}^{s_{j}}{ }^{-1}(w f) \zeta_{w s_{j}}\right) \\
& =\sum_{w \in \mathrm{W}(\nu)}\left(\Lambda_{w, w}^{s_{j}}{ }^{-1} \Lambda_{w}(w f)+\Lambda_{w, w s_{j}}^{s_{j}}{ }^{-1} \Lambda_{w}\left(w s_{j} f\right)\right) \Lambda_{w}^{-1} \zeta_{w} \\
& =\sum_{w \in \mathrm{W}(\nu)}\left(\prod_{a \in \Omega_{\nu_{j}, \nu_{j}}}\left(\chi_{j}(\nu)-\chi_{j+1}(\nu)+\hbar_{a}\right)\right) \frac{w s_{j} f-w f}{\chi_{w(j)}-\chi_{w(j+1)}} \Lambda_{w}^{-1} \zeta_{w} \\
& =\Psi_{\alpha}\left(\left(\prod_{a \in \Omega_{\nu_{j}, \nu_{j}}}\left(\chi_{j}(\nu)-\chi_{j+1}(\nu)+\hbar_{a}\right)\right) \frac{\left(s_{j} f\right)(\nu)-f(\nu)}{\chi_{j}(\nu)-\chi_{j+1}(\nu)}\right),
\end{aligned}
$$

which completes the proof.

Let $R(\alpha)$ be the Khovanov-Lauda-Rouquier algebra over the graded commutative ring $\mathbf{H}$ associated with the data $\left(\mathrm{A}, \mathrm{P}, \Pi, \Pi^{\vee}\right)$ and the polynomials $\mathcal{P}_{i}(u, v), \mathcal{Q}_{i, j}(u, v) \in \mathbf{H}[u, v]$ defined in (1.2.6). We take the $\mathbb{Z}$-grading defined by (1.2.7). Then we have a faithful graded polynomial representation $\operatorname{Pol}(\alpha)$ of $R(\alpha)$ given in Proposition 1.5.

Now we can state and prove the main result of this paper.

Theorem 2.8. There exists a unique $\mathbf{H}$-algebra isomorphism $\Theta: R(\alpha) \longrightarrow \mathfrak{R}(\alpha)$ such that

$$
\begin{aligned}
& \Theta(\mathrm{e}(\nu))=e(\nu), \quad \Theta\left(\mathrm{x}_{k}(\nu)\right)=\varkappa_{k}(\nu), \quad \Theta\left(\mathrm{r}_{t}(\nu)\right)=\tau_{t}(\nu) \\
& \quad \text { for } \nu \in I^{\alpha}, k=1, \ldots, m \text { and } t=1, \ldots, m-1 .
\end{aligned}
$$

Proof. We can easily identify $\operatorname{Pol}(\alpha)$ with $\mathfrak{P o l}(\alpha)$. It follows from (1.2.8), Proposition 1.5 and Proposition 2.7 that there exists a unique injective $\mathbf{H}$-algebra homomorphism $\Theta$ satisfying (2.3.15). By Lemma 2.3, $\mathfrak{R}(\alpha)$ is generated by $e(\nu), \varkappa_{k}(\nu)$ and $\tau_{t}(\nu)$, and hence $\Theta$ is surjective.

\section{IndeComposable PROJECTIVE MOdules AND LOWER GLOBAL BASES}

In this section, we give a 1-1 correspondence between Kashiwara's lower global basis (or Lusztig's canonical basis) of $U_{\mathbb{A}}^{-}(\mathfrak{g})$ (resp. $V_{\mathbb{A}}(\lambda)$ ) and the set of isomorphism classes of indecomposable projective graded $R$-modules (resp. indecomposable projective graded $R^{\lambda}$-modules) when any of the diagonal entries of the symmetric Borcherds-Cartan matrix $A=\left(a_{i j}\right)_{i, j \in I}$ does not vanish. Let us keep all the notations appeared in the previous sections. We first suppose that the symmetric Borcherds-Cartan matrix $A$ is arbitrary.

For a given quasi-projective variety $X$ over $\mathbb{C}$ with an action of a complex linear algebraic group $G$ and $A, B \in \mathbf{D}_{\mathrm{G}}^{b}(X)$, let $\operatorname{Hom}_{\mathrm{G}}^{k}(A, B)=\operatorname{Hom}_{\mathbf{D}_{\mathrm{G}}^{b}(X)}(A, B[k])$, where [.] is the 
translation functor. Recall the map $\pi_{\nu}$ given in (2.1.3). For $\nu \in I^{\alpha}$, we write

$$
\mathcal{L}_{\nu}=R \pi_{\nu !}\left(\mathbb{C}_{\widetilde{\mathcal{F}}_{\nu}}\left[2 \operatorname{dim}_{\mathbb{C}} \widetilde{\mathcal{F}}_{\nu}\right]\right) \in \mathbf{D}_{\mathrm{G}_{\alpha}^{\Omega}}^{b}\left(\mathcal{E}_{\alpha}\right) .
$$

Note that $\mathcal{L}_{\nu}$ is semisimple [11, Proposition 4.1]. Let $\mathcal{L}_{\alpha}=\bigoplus_{\nu \in I^{\alpha}} \mathcal{L}_{\nu}$. Then, by the same argument as in the proof of [5, Lemma 8.6.1], we obtain

$$
\mathfrak{R}(\alpha) \simeq \operatorname{Hom}_{\mathrm{G}_{\alpha}^{\Omega}}^{*}\left(\mathcal{L}_{\alpha}, \mathcal{L}_{\alpha}\right) \text { and } \mathfrak{R}_{\nu, \nu^{\prime}} \simeq \operatorname{Hom}_{\mathrm{G}_{\alpha}^{\Omega}}^{*}\left(\mathcal{L}_{\nu}, \mathcal{L}_{\nu^{\prime}}\right) \quad \text { for } \nu, \nu^{\prime} \in I^{\alpha} .
$$

Then $\mathfrak{R}(\alpha)$ is isomorphic to the opposite algebra $\operatorname{Hom}_{\mathrm{G}_{\alpha}^{\Omega}}^{*}\left(\mathcal{L}_{\alpha}, \mathcal{L}_{\alpha}\right)^{\text {op }}$ of $\operatorname{Hom}_{\mathrm{G}_{\alpha}^{\Omega}}^{*}\left(\mathcal{L}_{\alpha}, \mathcal{L}_{\alpha}\right)[5$, Section 8.6].

For $\alpha \in \mathrm{Q}^{+}$, let $\mathcal{P}_{\alpha}$ be the set of isomorphism classes of simple $\mathrm{G}_{\alpha}^{\Omega}$-equivariant perverse sheaves $\mathcal{L}$ on $\mathcal{E}_{\alpha}$ such that $\mathcal{L}[k]$ appears as a direct summand of $\mathcal{L}_{\alpha}$ for some $k \in \mathbb{Z}$. Let $\mathcal{Q}_{\alpha}$ be the full subcategory of $\mathbf{D}_{\mathrm{G}_{\alpha}^{\Omega}}^{b}\left(\mathcal{E}_{\alpha}\right)$ consisting of $\mathcal{L}$ having the form

$$
\mathcal{L} \simeq \mathcal{L}_{1}\left[k_{1}\right] \oplus \cdots \oplus \mathcal{L}_{r}\left[k_{r}\right]
$$

for some $\mathcal{L}_{i} \in \mathcal{P}_{\alpha}$ and $k_{i} \in \mathbb{Z}$. Then $\mathcal{L}_{\alpha}$ belongs to $\mathcal{Q}_{\alpha}$ by the decomposition theorem [1].

We now identify $R(\alpha)$ with $\mathfrak{R}(\alpha)$ via Theorem 2.8. Then, for $\mathcal{L} \in \mathcal{Q}_{\alpha}$, the vector space $\operatorname{Hom}_{\mathrm{G}_{\alpha}^{\Omega}}^{*}\left(\mathcal{L}_{\alpha}, \mathcal{L}\right)$ has the left $R(\alpha)$-module structure. Moreover, $\operatorname{Hom}_{\mathrm{G}_{\alpha}^{\Omega}}^{*}\left(\mathcal{L}_{\alpha}, \mathcal{L}\right)$ is a projective $R(\alpha)$-module by the construction of $\mathcal{Q}_{\alpha}$. Then we obtain the following proposition by general results on idempotent complete categories.

Proposition 3.1. Let

$$
\Upsilon_{\alpha}: \mathcal{Q}_{\alpha} \longrightarrow R(\alpha) \text {-pmod } \text { and } \Xi_{\alpha}: R(\alpha) \text {-pmod } \longrightarrow \mathcal{Q}_{\alpha}
$$

be the functors given by

$$
\Upsilon_{\alpha}(\mathcal{L})=\operatorname{Hom}_{\mathrm{G}_{\alpha}^{\Omega}}^{*}\left(\mathcal{L}_{\alpha}, \mathcal{L}\right) \quad \text { and } \quad \Xi_{\alpha}(P)=\mathcal{L}_{\alpha} \otimes_{R(\alpha)} P
$$

for $\mathcal{L} \in \mathcal{Q}_{\alpha}$ and $P \in R(\alpha)$-pmod, respectively.

Then $\Upsilon_{\alpha}$ is an equivalence of categories and $\Xi_{\alpha}$ is its quasi-inverse.

From now on, we assume that $a_{i i} \neq 0$ for any $i \in I$. Let $K\left(\mathcal{Q}_{\alpha}\right)$ denote the $\mathbb{A}$-module generated by $[\mathcal{L}]$ for $\mathcal{L} \in \mathcal{Q}_{\alpha}$ subject to the relations $\left[\mathcal{L}_{1} \oplus \mathcal{L}_{2}\right]=\left[\mathcal{L}_{1}\right]+\left[\mathcal{L}_{2}\right]$ and $[\mathcal{L}[k]]=q^{-k}[\mathcal{L}]$ for $k \in \mathbb{Z}$.

Let

$$
\mathcal{U}_{\mathbb{A}}^{-}=\bigoplus_{\alpha \in Q^{+}} K\left(\mathcal{Q}_{\alpha}\right)
$$

Then, together with the induction and restriction functors given in $[11,17], \mathcal{U}_{\mathbb{A}}^{-}$becomes an $\mathbb{A}$-bialgebra and there exists an isomorphism [11, Section 5]

$$
\Psi: U_{\mathbb{A}}^{-}(\mathfrak{g}) \stackrel{\sim}{\longrightarrow} \mathcal{U}_{\mathbb{A}}^{-},
$$


where $U_{\mathbb{A}}^{-}(\mathfrak{g})$ is the $\mathbb{A}$-form of the negative half of the quantum group $U_{q}(\mathfrak{g})$. Set

$$
\mathbf{B}=\bigsqcup_{\alpha \in \mathbf{Q}^{+}} \Psi^{-1}\left(\mathcal{P}_{\alpha}\right) .
$$

The set $\mathbf{B}$ coincides with the lower global basis (or the canonical basis) of $U_{\mathbb{A}}^{-}(\mathfrak{g})$.

Let $K_{0}(R)=\bigoplus_{\alpha \in \mathrm{Q}^{+}} K_{0}(R(\alpha)$-pmod) be the Grothendieck group given in (1.2.3) and define

$$
\Upsilon=\bigsqcup_{\alpha \in Q^{+}} \Upsilon_{\alpha}: \mathcal{U}_{\mathbb{A}}^{-} \stackrel{\sim}{\longrightarrow} K_{0}(R),
$$

where $\Upsilon_{\alpha}$ is the isomorphism induced by Proposition 3.1. Using the same argument as in $\left[20\right.$, Section 4.6], it follows from Theorem 1.3 and (3.1.17) that the isomorphism $\Phi: U_{\mathbb{A}}^{-}(\mathfrak{g}) \rightarrow$ $K_{0}(R)$ given in Theorem 1.3 satisfies

$$
\Phi=\Upsilon \circ \Psi
$$

Consequently, the first application of our main result follows.

Theorem 3.2. The isomorphism $\Phi$ gives a 1-1 correspondence between $\mathbf{B}$ and the set of isomorphism classes of indecomposable projective $R$-modules.

Let $\lambda$ be a dominant integral weight in $\mathrm{P}^{+}$and $K_{0}\left(R^{\lambda}\right)=\bigoplus_{\alpha \in \mathrm{Q}^{+}} K_{0}\left(R^{\lambda}(\alpha)\right.$-pmod) be the Grothendieck group given in (1.2.3). Let $v_{\lambda}$ be the highest weight vector of $V_{\mathbb{A}}(\lambda)$ and define $\mathbf{p}_{\lambda}: U_{\mathbb{A}}^{-}(\mathfrak{g}) \longrightarrow V_{\mathbb{A}}(\lambda)$ by

$$
\mathbf{p}_{\lambda}(x)=x v_{\lambda}
$$

for $x \in U_{\mathbb{A}}^{-}(\mathfrak{g})$. Similarly, we define $\mathbf{q}_{\lambda}: K_{0}(R) \longrightarrow K_{0}\left(R^{\lambda}\right)$ by

$$
\mathbf{q}_{\lambda}(P)=R^{\lambda}(\alpha) \otimes_{R(\alpha)} P
$$

for $P \in R(\alpha)$-pmod. Then we have the following commutative diagram:

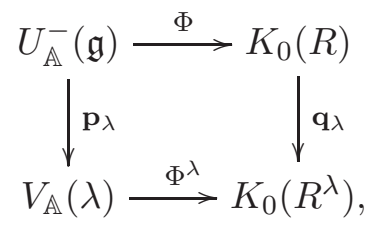

where $\Phi^{\lambda}: V_{\mathbb{A}}(\lambda) \rightarrow K_{0}\left(R^{\lambda}\right)$ is the isomorphism given in Theorem 1.4. Then $\mathbf{B}^{\lambda}:=\mathbf{p}_{\lambda}(\mathbf{B}) \backslash\{0\}$ coincides with the lower global basis (or the canonical basis) of $V_{\mathbb{A}}(\lambda)$. For $P \in R(\alpha)$-pmod, since $R^{\lambda}(\alpha) \otimes_{R(\alpha)} P$ can be viewed as an $R(\alpha)$-module, we have a surjective $R(\alpha)$-module homomorphism

$$
P \rightarrow R^{\lambda}(\alpha) \otimes_{R(\alpha)} P
$$

which implies that $\mathbf{q}_{\lambda}$ takes an indecomposable projective $R(\alpha)$-module to an indecomposable projective $R^{\lambda}(\alpha)$-module or 0 . Therefore we obtain the second application of our main result. 
Corollary 3.3. The isomorphism $\Phi^{\lambda}$ gives a 1-1 correspondence between $\mathbf{B}^{\lambda}$ and the set of isomorphism classes of indecomposable projective $R^{\lambda}$-modules.

\section{REFERENCES}

1. A. A. Beilinson, J. Bernstein, and P. Deligne, Faisceaux pervers, Astérisque, 100, Soc. Math. France, Paris, 1982.

2. J. Bernstein and V. Lunts, Equivariant Sheaves and Functors, Lecture Notes in Math. 1578, SpringerVerlag, Berlin, 1994.

3. M. Brion, Equivariant cohomology and equivariant intersection theory, Notes by Alvaro Rittatore. NATO Adv. Sci. Inst. Ser. C Math. Phys. Sci., 514, Representation theories and algebraic geometry (Montreal, PQ, 1997), 1-37, Kluwer Acad. Publ., Dordrecht, 1998.

4. Lectures on the geometry of flag varieties, Topics in cohomological studies of algebraic varieties, 33-85, Trends Math., Birkhäuser, Basel, 2005.

5. N. Chriss and V. Ginzburg, Representation Theory and Complex Geometry, Birkhäuser Boston, Inc., Boston, MA, 1997.

6. W. Fulton, Equivariant cohomology in algebraic geometry, Eilenberg lectures, Columbia University, Spring 2007.

7. K. Jeong, S.-J. Kang, and M. Kashiwara, Crystal bases for quantum generalized Kac-Moody algebras, Proc. London Math. Soc. (3) 90 (2005), no. 2, 395-438.

8. S.-J. Kang and M. Kashiwara, Categorification of Highest Weight Modules via Khovanov-Lauda-Rouquier Algebras, to appear in Invent. Math.; arXiv:1102.4677 (2011).

9. S.-J. Kang, M. Kashiwara, and S.-j. Oh, Categorification of highest weight modules over quantum generalized Kac-Moody algebras, to appear in Mosc. Math. J.; arXiv:1106.2635 (2011).

10. S.-J. Kang, S.-j. Oh, and E. Park, Categorification of quantum generalized Kac-Moody algebras and crystal bases, to appear in Internat. J. Math.; arXiv:1102.5165v3 (2011).

11. S.-J. Kang and O. Schiffmann, Canonical bases for quantum generalized Kac-Moody algebras, Adv. Math. 200 (2006), no. 2, 455-478.

12. M. Kashiwara, On crystal bases of the q-analogue of universal enveloping algebras, Duke Math. J. 63 (1991), no. 2, 465-516.

13. M. Khovanov and A. Lauda, A diagrammatic approach to categorification of quantum groups I, Represent. Theory 13 (2009), 309-347.

14. A A diagrammatic approach to categorification of quantum groups II, Trans. Amer. Math. Soc. 363 (2011), no. 5, 2685-2700.

15. G. Lusztig, Canonical bases arising from quantized enveloping algebras, J. Amer. Math. Soc. 3 (1990), no. 2, 447-498.

16. _ Quivers, perverse sheaves, and quantized enveloping algebras, J. Amer. Math. Soc. 4 (1991), no. 2, $365-421$.

17. Introduction to Quantum Groups, Progress in Mathematics, 110. Birkhäuser Boston, Inc., Boston, MA, 1993.

18. R. Rouquier, 2 Kac-Moody algebras, arXiv:0812.5023 (2008).

19. __ Quiver Hecke algebras and 2-Lie algebras, Algebra Colloq. 19 (2012), no. 2, 359-410. 
20. M. Varagnolo and E. Vasserot, Canonical bases and KLR algebras, J. Reine Angew. Math. 659 (2011), 67-100.

21. B. Webster, Knot invariants and higher dimensional representation theory I: diagrammatic and geometric categorification of tensor products, arXiv:1001.2020.

Department of Mathematical Sciences and Research Institute of Mathematics, Seoul National University, 599 Gwanak-ro, GwanaK-Gu, Seoul 151-747, Korea

E-mail address: sjkang@snu.ac.kr

Research Institute for Mathematical Sciences, Kyoto University, Kyoto 606-8502, Japan And Department of Mathematical Sciences, Seoul National University, 599 Gwanakro, Gwanak-Gu, SEOUl 151-747, KOREA

E-mail address: masaki@kurims.kyoto-u.ac.jp

Department of Pure and Applied Mathematics, Graduate School of Information Science and Technology, Osaka University, Toyonaka, Osaka 560-0043, Japan

E-mail address: pwy@cr.math.sci.osaka-u.ac.jp 